\title{
Further development in theory/data closure of the photoelectron-driven polar wind and day-night transition of the outflow
}

\author{
S. W. Y. Tam, F. Yasseen, T. Chang \\ Center for Space Research, Massachusetts Institute of Technology, Cambridge, MA 02139, USA
}

Received: 9 June 1997 / Revised: 9 January 1998 / Accepted: 24 February 1998.

\begin{abstract}
Recent in situ observations have revealed novel features in the polar wind. Measurements between 5000 and $9000 \mathrm{~km}$ altitude by the Akebono satellite indicate that both $\mathrm{H}^{+}$and $\mathrm{O}^{+}$ions can have remarkably higher outflow velocities in the sunlit region than on the nightside. Electrons also display an asymmetric behavior: the dayside difference in energy spread, greater for upward-moving than downward-moving electrons, is absent on the nightside. Here, we discuss the further development of a theory by Tam et al. that can explain most of these observed peculiar properties by properly taking into account the global, kinetic, collisional effects of the sunlit photoelectrons, on the background polar wind and the electric field. Quantitative comparisons of the calculated results with actual data will be described. In addition, transition from the daytime photoelectrondriven polar wind to the night-time polar wind will be discussed.
\end{abstract}

\section{Introduction}

Plasma flows along magnetic field lines are a very common occurrence in space plasmas. In particular, the existence of an outflow of plasma along the open magnetic field lines emanating from the polar region of the ionosphere was first proposed by Axford (1968) and Banks and Holzer (1968). This outflow was termed "polar wind" in analogy with the solar wind, which had been recently observed (Parker, 1958). These early studies included the ambipolar electric field among the mechanisms governing the plasma outflow, and suggested that the field arises from the separation between the ions and the electrons due to their mass difference. Because the polar cap, in general, is a relatively

Correspondence to: $\mathrm{S}$. Tam quiescent region, the ambipolar effect is a major contribution to the electric field in the "classical" polar wind, the steady-state, quasi-neutral, current-free outflow of plasma above the polar cap.

The ambipolar field itself exists self-consistently with the background plasma. It is therefore influenced by other mechanisms that govern the dynamics of the particles and that may need to be included in the description. For example, the geomagnetic field, which decreases with altitude, gives rise to the mirror force that changes the particles' pitch angles. Coulomb interactions among all the species lead to energy exchange and pitch angle diffusion. These effects are essential to the dynamics of the particles, and therefore, can affect the polar wind electric field.

Recent observations have suggested that another contribution, photoelectron populations generated in the sunlit ionosphere, can alter the polar wind significantly. Our study on the photoelectron-driven polar wind is motivated by these increasingly convincing experimental indications.

This study is structured as follows: in the remainder of this section, we will discuss in more detail the experimental evidence motivating our study, and why traditional approaches to polar wind modeling are inadequate to describe this experimental evidence. In Sect. 2, we shall discuss the physics of photoelectrons, and how they can affect the polar wind dynamics. In Sect. 3, the self-consistent hybrid model on which our calculations are based will be described. In Sect. 4, results generated from the model will be compared with observations to demonstrate the development in theory/ data closure. In addition, the day-night transition of the photoelectron-driven polar wind will be discussed in terms of results from a parametric study.

\subsection{Motivation}

Early polar cap measurements obtained by the ISIS-1 satellite showed evidence of "anomalous" field-aligned photoelectron fluxes in both upward and downward 
directions, where the downgoing (return) fluxes were considerably smaller than the outgoing fluxes above a certain energy (Winningham and Heikkila, 1974). Such non-thermal features were later confirmed with better resolution by the DE-1 and -2 satellites (Winningham and Gurgiolo, 1982): outgoing field-aligned electron fluxes in the photoelectron energy range were observed by HAPI (High Altitude Plasma Instrument) on DE-1 and LAPI (Low Altitude Plasma Instrument) on DE-2; evidence of downstreaming electron fluxes was also found in the low-altitude distribution measured by LAPI. These fluxes are considered anomalous because their existence cannot be related to the idea of thermal conductivity and temperature gradient-induced fluxes in classical fluid theories. Similar to the ISIS-1 measurements, the return fluxes observed by DE-2 were comparable to the outgoing fluxes below some truncation energy, but considerably smaller above that. As suggested by Winningham and Gurgiolo (1982), the existence of such downstreaming fluxes may be due to reflection of electrons by the ambipolar electric field along the geomagnetic field line above the satellite. The truncation energy, obtained by comparing the outgoing and the return electron fluxes, would thus provide an estimate for the potential drop due to the electric field. These authors observed that this truncation energy ranged from 5 to $60 \mathrm{eV}$, and thus were able to deduce the magnitude of the potential drop above the altitude of the satellite $(\sim 500 \mathrm{~km})$. Unfortunately, existing classical polar wind theories can only account for a much smaller potential drop (Ganguli, 1996, and references therein). Winningham and Gurgiolo (1982) also pointed out that variation of the truncation energy was due to changes in the solar zenith angle at the production layer below the satellite. The solar zenith angle is related to the photoionization rate, which itself is related to the local ionospheric photoelectron density (Jasperse, 1981). These observations therefore imply a causal relationship between the local photoelectron density below the satellite and the potential drop along the field line above it, and are consistent with the idea that the photoelectrons may significantly affect the ambipolar electric field.

While the observations discussed above have implied that photoelectrons may contribute to the dynamics of the polar wind, more recent evidence indicates that the polar wind characteristics themselves are affected by the photoelectrons. In situ measurements by the Akebono satellite have revealed novel features in the polar wind: day-night asymmetries in the ion and electron features. The most dramatic are asymmetries in the ion outflow velocities (Abe et al., 1993a): satellite data between 5000 and $9000 \mathrm{~km}$ altitude have indicated remarkably higher outflow velocities for the major ion species, $\mathrm{H}^{+}$and $\mathrm{O}^{+}$, in the sunlit region than on the nightside. For example, the $\mathrm{H}^{+}$velocity $\left(u_{h}\right)$ was found to be about $12 \mathrm{~km} / \mathrm{s}$ on the dayside, but only about $5 \mathrm{~km} / \mathrm{s}$ on the nightside. Similarly, the $\mathrm{O}^{+}$velocity $\left(u_{o}\right)$ in the sunlit region $(\sim 7 \mathrm{~km} / \mathrm{s})$ is about twice that in the midnight sector $(\sim 3 \mathrm{~km} / \mathrm{s})$. A day-night asymmetry was also observed in the electron behavior. The instrument distinguished between electrons according to their velocities along the geomagnetic field line. On the dayside, it was found that the temperature of the upstreaming population is greater than that of the downstreaming population, i.e., $T_{e, \text { up }}>T_{e, \text { down }}$, indicative of an upwardly directed heat flux (Yau et al., 1995). On the nightside, in contrast, no such up-down anisotropy was observed (Abe et al., 1996). The observed polar wind day-night asymmetries are summarized in Table 1.

Besides the day-night asymmetries, Akebono measurements between 5000 and $9000 \mathrm{~km}$ altitude have also revealed other, sometimes unexpected, ion transport properties in the polar region (Abe et al., 1993a). For example, $\mathrm{O}^{+}$was most often found to be the major ion species, dominant over $\mathrm{H}^{+}$, contrary to the traditional belief that due to their heavier mass very few $\mathrm{O}^{+}$ions are able to overcome the gravitational force and escape to such high altitudes. The measured outflow velocities for both the $\mathrm{H}^{+}$and $\mathrm{O}^{+}$ions in general increase monotonically with altitude, and the flows for both species are supersonic at high altitudes. In fact, the measured $\mathrm{O}^{+}$ outflow velocities (see earlier) are much larger than the values expected from classical polar wind models (e.g. Schunk and Watkins, 1981). All these ion outflow characteristics, particularly the enhanced ion outflow velocities, suggest a higher ambipolar electric field than that predicted by classical polar wind models (Ganguli, 1996, and references therein), and are consistent with the values of the field-aligned potential drop deduced by Winningham and Gurgiolo (1982) based on the DE-2 measurements.

Because of the marked day-night asymmetries observed in several characteristics of the polar wind, and the fact that photoelectrons exist primarily in the sunlit ionosphere, they are the natural candidate to account for the day-night asymmetries. Indeed, collisionless kinetic calculations by Lemaire (1972) showed that escaping photoelectrons may enhance the electric field and increase the ion outflow velocities in the polar wind. Photoelectrons, therefore, may provide a possible unifying explanation for the observations of both sets of satellites, the magnitude of the ambipolar electric field deduced from the DE-2 measurements, and the daynight asymmetries and enhanced ion outflow velocities observed by the Akebono satellite. Because Coulomb collisions may also influence the dynamics of the photoelectrons, for example, by transferring their energy to other particle components in the polar wind, and thereby reducing the escaping photoelectron flux,

Table 1. A summary of the polar wind day-night asymmetries observed by the Akebono satelite. The average ion outflow velocities were measured between 5000 and $9000 \mathrm{~km}$ altitude, and the electron temperature ratios were obtained at about 1700 $\mathrm{km}$ altitude

\begin{tabular}{lll}
\hline & Day & Night \\
\hline$u_{h}$ & $12-13 \mathrm{~km} / \mathrm{s}$ & $4-5 \mathrm{~km} / \mathrm{s}$ \\
$u_{o}$ & $6-7 \mathrm{~km} / \mathrm{s}$ & $2-3 \mathrm{~km} / \mathrm{s}$ \\
$T_{e}$, up $/ T_{e}$, down & $1.5-2$ & $\approx 1$ \\
\hline
\end{tabular}


collisional effects should also be taken into account in determining the impact of photoelectrons on the electric field. Our goal, therefore, is to address these observations quantitatively by incorporating the complete photoelectron physics into a self-consistent, global description of the polar wind.

\subsection{Traditional approaches in polar wind modeling}

Let us begin by listing some criteria that will enable us to define the problem more precisely. First, we will consider the polar wind only at altitudes above 500 $\mathrm{km}$ (which corresponds roughly to the polar orbits of DE-2). At such altitudes, neutral densities are low enough to neglect the "chemical" reactions such as photoionization, recombination, etc. Second, the magnitude of the geomagnetic field is such that the gyration period and Larmor radius, for all particle species, are much smaller than any relevant time or length scales. We can therefore use the guiding center approximation. Third, the gradients of the geomagnetic field are such that only transport along the geomagnetic field line is important. The time-dependent distribution function $f\left(t, s, v_{\|}, v_{\perp}\right)$ for a given particle species is therefore governed by the following collisional gyrokinetic equation:

$\left[\frac{\partial}{\partial t}+v_{\|} \frac{\partial}{\partial s}-\left(g-\frac{q_{c}}{m} E_{\|}\right) \frac{\partial}{\partial v_{\|}}-v_{\perp}^{2} \frac{B^{\prime}}{2 B}\left(\frac{\partial}{\partial v_{\|}}-\frac{v_{\|}}{v_{\perp}} \frac{\partial}{\partial v_{\perp}}\right)\right] f$

$=\frac{\delta f}{\delta t}=C f$,

where $s$ is the distance along the magnetic field line $B, q_{c}$ and $m$ are the algebraic electric charge and mass of the species respectively, $E_{\|}$is the field-aligned electric field, $g$ is the gravitational acceleration, $B^{\prime} \equiv \mathrm{d} B / \mathrm{d} s, \delta f / \delta t$ represents the rate of change of the distribution function due to collisions, and $C$ is a collisional operator for Coulomb interaction, which is the dominant type of collision above $300 \mathrm{~km}$ altitude. Equation (1) includes the major forces a particle experiences as it travels along the geomagnetic field line: gravitational force, fieldaligned electric force, mirror force, and forces that are due to Coulomb collisions.

Obviously, solutions based on Eq. (1), even with additional simplifications (e.g. steady-state, quasi-neutral, current-free outflow of electrons and $\mathrm{O}^{+}$and $\mathrm{H}^{+}$ ions, conditions satisfied by the "classical" polar wind), are difficult to obtain. Further approximations thus seem necessary. Traditionally, there have been two schools of thought in polar wind modeling, collisionless kinetic calculations (e.g. Lemaire, 1972) and moment equations (e.g. Banks and Holzer, 1968; Schunk, 1977), each based on a different type of approximation to the kinetic, collisional approach embodied in Eq. (1).

In collisionless kinetic calculations, one neglects the Coulomb collisional term in Eq. (1), leading to the wellknown Vlasov equation. In the steady state, exact solution of the distribution function in the resulting collisionless equation can be obtained by relating the independent variables $\left(s, v_{\|}\right.$, and $\left.v_{\perp}\right)$ through the particles' constants of motion (energy and magnetic moment). The resulting distribution function for each particle species, however, is a function also of the electric potential. To determine the electric potential, the local quasi-neutrality and current-free conditions can be used.

The collisionless kinetic approximation is valid at high altitudes (roughly $>3000 \mathrm{~km}$ ). One should bear in mind, however, that the cumulative effect of even weak Coulomb collisions may significantly affect the distribution function as the plasma flows over large distances. Near the polar wind source, the Coulomb collisional effect is definitely non-negligible. In order to take this effect into account, some investigators thus resort to the moment approach.

Moment-based models describe the particle transport using a few variables to characterize the species, such as density, velocity, temperature and heat fluxes. The equations that describe these variables are obtained by taking velocity moments of Eq. (1). Collisional effects are represented in these equations by the corresponding velocity moments of the collisional term in Eq. (1). Consequently, the collisional effects on one species due to another are described by a velocity-averaged collisional frequency, which depends on the density, velocity and temperature moments of both species.

However, due to the convective term $v_{\|} \partial / \partial_{s}$ in Eq. (1), each of these equations contains a velocitymoment term of the next higher order. Therefore, an assumption needs to be made in order to close the system of equations. Depending on the assumption, the system may consist of a different number of moments. Existing models that have been discussed in the literature consist of five, eight, ten, thirteen, sixteen, and even twenty moments (Schunk, 1977). A variety of these moment-based models has been applied to the classical polar wind to take into account collisional effects (e.g. Banks and Holzer, 1968; Raitt et al., 1975; Schunk and Watkins, 1981) and even anisotropies (e.g. Ganguli et al., 1987; Demars and Schunk, 1989; Blelly and Schunk, 1993).

Because of the complexity and non-linear nature of the system of moment equations, analytic solutions are hard to achieve. Therefore, moment-based models are generally solved numerically. Because of the intrinsically stiff nature of these systems of moment equations (due essentially to the high mass ratios), even numerical solutions are not straightforward to obtain. In addition, these systems exhibit a number of singularities that arise from the assumption used to close the system (Yasseen and Retterer, 1991). In the polar wind case, for example, the steady-state sixteen-moment model exhibits singularities near the ion sonic points, i.e., the point at which the ion's thermal speed corresponds to its flow velocity, and cannot provide transonic solutions easily. However, such transonic solutions are required for the appropriate description of the polar wind because observations indicate that both the $\mathrm{H}^{+}$and $\mathrm{O}^{+}$outflows are supersonic at high altitudes (Abe et al., 1993a). In order to circumvent this difficulty, some investigators seek the 
steady-state solution by solving a time-dependent problem until a steady state is reached (Blelly and Schunk, 1993).

Implicit in the two complementary approaches outlined above is the assumption of the existence of a transition region separating a low-altitude collisional region where the flow is subsonic and a high-altitude collisionless region where the flow is supersonic. The simplest way to combine these two approaches is to introduce the concept of a baropause, the altitude at which the average collisional mean free path corresponds to the scale length of the outflow. The moment equations are then solved from a set of low-altitude boundary conditions up to the baropause. The resulting moments at the baropause thus provide the boundary conditions for the collisionless kinetic calculations that will be applied to higher altitudes (Barakat and Schunk, 1983).

However, moment-based models suffer from an intrinsic limitation that is incompatible with the inherent nature of Coulomb collisions. On the one hand, different species are characterized by different collisional mean free paths and scale lengths, and it is not clear that the transition from collisional to collisionless for all species present will occur at the same altitudes. However, these models assume that the distribution functions are close to local thermodynamic equilibrium, thus describing the collisions using a velocity-averaged collisional frequency which depends on the local densities, velocities and temperatures of the plasma components. However, it is well known that the Coulomb collisional cross section for a charged particle is strongly dependent on its velocity relative to the background (Ichimaru, 1986). In general, the higher this velocity, the smaller the cross section and collisional frequency, and the longer the collisional mean free path. Energetic particles therefore have smaller collisional frequency and longer collisional mean free path than the corresponding characteristic values used in the moment equations. Thus, the presence of energetic particles can limit the applicability of moment equations and, by the same token, invalidate the concept of a baropause. We can clearly see now why the moment approach cannot be adequately used to include the collisional physics of the photoelectrons in a unified description of the polar wind. Instead, a global, kinetic approach is necessary. In this study, a "global" approach means an evolutionary model involving crossscale interactions. Thus, by "global kinetic" here, we mean the ability of the polar wind model to resolve the mesoscale evolution of the particle distribution function resulting from microscale interactions.

A global, kinetic, collisional description of photoelectrons in a self-consistent polar wind picture was first achieved by Tam et al. (1995a). Results generated from their model successfully addressed the polar wind qualitative features observed by the polar-orbiting satellites (Winningham and Gurgiolo, 1982; Abe et al., 1993a, b), and demonstrated that the ambipolar electric field arises due to the anisotropy of the particle distributions in an inhomogeneous magnetic field. In this study, we shall discuss in more detail the role of photoelectrons in the day-night transition of the polar wind, and their impact on the outflow based on new results generated from the self-consistent model. In addition, we shall present a new polar wind solution that is quantitatively more similar to the Akebono observations, thus improving our earlier qualitative results.

\section{Photoelectrons, energy fluxes and electric field}

The impact of photoelectrons on the polar wind was postulated in the early literature. Axford (1968) proposed that the charge separation enhanced by the escaping polar wind photoelectron flux might increase the electric field which, in turn, might accelerate the ions. Indeed, results of a collisionless kinetic calculation by Lemaire (1972) agreed with Axford's postulation. The formulation of the calculation took into account the kinetic effects of polar wind photoelectrons on the ambipolar electric field; the escaping photoelectron flux was shown to be a possible mechanism to enhance the electric field, and by which to accelerate the ions.

The global kinetic collisional physics of suprathermal electrons in a steady-state space plasma outflow was first considered by Scudder and Olbert (1979) in their study of the solar wind halo electrons. These authors related the anomalous field-aligned electron heat fluxes observed in the solar wind to the non-local nature of the electron distributions, and demonstrated the formation of such non-thermal features using a simplified collisional operator. They also suggested that these suprathermal electrons, through their anomalous contribution to the energy flux, may significantly increase the ambipolar electric field along the magnetic field lines, thereby "driving" the solar wind (Olbert, 1982).

An analogous situation exists for the dayside, photoelectron-driven polar wind, as demonstrated by more recent collisional calculations. It has been shown by Yasseen et al. (1989) that the polar wind photoelectrons can give rise to the non-thermal distributions observed by the DE satellites (Winningham and Gurgiolo, 1982). The effect on the polar wind due to the energy fluxes associated with these photoelectrons has been examined by Tam et al. (1995b), who concluded that such anomalous electron energy fluxes may significantly increase the ambipolar electric field.

The impact of photoelectron energy fluxes on the polar wind dynamics can be understood heuristically in a simplified scenario: the high-altitude (collisionless) "classical" (steady-state) polar wind. In this scenario, every particle species must satisfy energy conservation:

$\frac{\partial}{\partial s}\left\{\frac{1}{B}\left[Q_{w}+n u\left(m \Phi_{G}+q_{c} \Phi_{E}\right)\right]\right\}=0$

where $n, u$, and $Q_{w}=\int_{u} \mathrm{~d} \mathbf{v} \frac{1}{2} m v^{2} v_{\|} f\left(s, v_{\|}, v_{\perp}\right)$ are respectively the density, velocity, and energy flux of the species, $\Phi_{G}$ is the gravitational potential, and $\Phi_{E}$ is the potential for the field-aligned electric field. Note that the energy flux, $Q_{w}$, is not the same as the heat flux, $Q=\int \mathrm{d} \mathbf{v} \frac{1}{2} m(\mathbf{v}-\mathbf{u})^{2}\left(v_{\|}-u\right) f\left(s, v_{\|}, v_{\perp}\right)$, which is a quantity more popularly used in the literature for the 
polar wind. The heat flux moment is taken about the mean velocity of the distribution function, while the energy flux is taken about the zero point in the velocity space. (The two fluxes are related in the following way: $Q_{w}=Q+n u\left(\frac{3}{2} T_{\|}+T_{\perp}+\frac{m u^{2}}{2}\right)$, where $T_{\|, \perp}$ are the parallel and perpendicular temperatures of the species.) Equation (2) is obtained by taking the energy moment of Eq. (1), and must be satisfied by every particle species. It illustrates a direct relation between the particle energy flux and the electric potential. The equations for different species are coupled through the ambipolar electric potential terms and the two classical polar wind constraints, quasi-neutrality and current-free flow. However, one can show from the electron equation that in an outflowing plasma, a larger upward electron energy flux generally leads to a larger electric potential drop.

Because photoelectrons carry a large amount of upward energy flux in the polar outflow, their presence, primarily in the sunlit ionosphere, thus enhances the dayside ambipolar electric field, thereby increasing the ion outflow velocities on the dayside. Photoelectrons, with their associated energy fluxes, can therefore provide not only a mechanism for the enhanced ion outflow velocities observed by the Akebono satellite (Abe et al., 1993a, b), but also the explanation for the observed daynight asymmetric ion and electron features in the polar wind (Abe et al., 1993a, 1996; Yau et al., 1995).

\section{Self-consistent hybrid model}

Recently, a self-consistent hybrid model has been developed by Tam et al. (1995a) to take into account the global kinetic collisional nature of the polar wind physics introduced by the photoelectrons. Our results in this study (which we present in Sect. 4) are based on the model. The model applies kinetic collisional description to the ions and the photoelectrons in a self-consistent model, thus enabling us to achieve two breakthroughs in polar wind modeling. One, it was the first to successfully incorporate the global kinetic collisional photoelectron effects into a self-consistent polar wind description. Two, due to its treatment of the ions, which we shall discuss later, the model was the first to generate selfconsistent global polar wind calculations whose solutions span continuously from a collisional subsonic regime at low altitudes to a collisionless supersonic regime at high altitudes.

The model is hybrid in that it consists of a kinetic and a fluid component. Photoelectrons (treated as test particles because of their low relative density) and both the $\mathrm{H}^{+}$and $\mathrm{O}^{+}$ions are described using a global kinetic collisional approach while thermal electron properties are determined from a simpler, fluid approach that also calculates the self-consistent ambipolar electric field. Because of its treatment of the thermal electrons, the model should be distinguished from traditional hybrid approaches where electrons are treated as a massless neutralizing fluid. The model is based on an iterative scheme combining the kinetic and fluid calcu- lations, that should converge to physically meaningful solutions.

\subsection{Fluid component}

In the formulation of the fluid calculation, the thermal electrons are assumed to be in a drifting Maxwellian distribution. This distribution, combined with the photoelectron results obtained from the kinetic component of the model, would form the total electron distribution. One of the purposes of the fluid calculation is to determine the various parameters that constitute the Maxwellian distribution.

The density and drift velocity of the thermal electrons are determined from the quasi-neutral and current-free constraints characterizing the classical polar wind, with input of densities and flow velocities of the other particle components $\left(\mathrm{H}^{+}, \mathrm{O}^{+}\right.$and photoelectrons) obtained from the kinetic part of the model. In particular, the current-free condition is:

$n_{e} u_{e}+n_{s} u_{s}=n_{h} u_{h}+n_{o} u_{o}$,

where the subscripts $e, s, h$, and $o$ stand for the thermal electron, suprathermal electron (photoelectron), $\mathrm{H}^{+}$, and $\mathrm{O}^{+}$species respectively. The density and drift velocity profiles of the thermal electrons (and in fact, of every plasma component) should also satisfy the continuity equation:

$\frac{\partial}{\partial s}\left(\frac{n u}{B}\right)=0$.

However, note that the current-free condition is consistent with the continuity equation. As will be discussed in the next section, the profiles of the particle components obtained from the kinetic part of the model have already satisfied Eq. (4). Therefore, by imposing the current-free condition throughout the whole simulation range, the resulting thermal electron density and velocity profiles must also satisfy Eq. (4).

The thermal electron temperature, $T_{e}$, together with the ambipolar electric field, are obtained from the moment equations of the next two higher orders, i.e. the momentum and energy transfer equations for the whole electron population:

$$
\begin{gathered}
B \frac{\partial}{\partial s}\left(\frac{n_{e} T_{e}+n_{e} m_{e} u_{e}^{2}+n_{s} T_{s \|}+n_{s} m_{e} u_{s}^{2}}{B}\right)+\left(n_{e}+n_{s}\right) \\
\left(m_{e} \frac{\partial \Phi_{G}}{\partial s}-e \frac{\partial \Phi_{E}}{\partial s}\right)+\frac{B^{\prime}}{B}\left(n_{e} T_{e}+n_{s} T_{s \perp}\right) \\
=\frac{\delta M_{e}}{\delta t}+\frac{\delta M_{s}}{\delta t}, \\
B \frac{\partial}{\partial s}\left[\frac{n_{e} u_{e}}{B}\left(\frac{5}{2} T_{e}+\frac{m_{e} u_{e}^{2}}{2}\right)+\frac{Q_{w s}}{B}+\left(\frac{n_{e} u_{e}}{B}+\frac{n_{s} u_{s}}{B}\right)\right. \\
\left.\left(m_{e} \Phi_{G}-e \Phi_{E}\right)\right]=\frac{\delta \mathscr{E}_{e}}{\delta t}+\frac{\delta \mathscr{E}_{s}}{\delta t}
\end{gathered}
$$

where $e$ is the unit charge, and $\delta M_{e, s} / \delta t$ and $\delta \mathscr{E}_{e, s} / \delta t$ represent the rates of momentum and energy transfers to the thermal and suprathermal electrons due to Coulomb 
collisions. These collisional transfer values are determined by the kinetic component of the model, based on momentum and energy conservation resulting from the symmetries of the Coulomb collisional operator (Ichimaru, 1986).

\subsection{Kinetic component}

The kinetic calculation is based on the global kinetic collisional test-particle model used in Yasseen et al. (1989) and Tam et al. (1995b). The model, based on a Monte Carlo technique, was adapted from a model originally developed by Retterer et al. (1987) for wave-particle interactions. In this self-consistent hybrid technique, kinetic calculations are used to describe the photoelectrons, and all the ions $\left(\mathrm{H}^{+}\right.$and $\left.\mathrm{O}^{+}\right)$. The densities of these particle species are normalized by Eq. (4).

Photoelectrons, due to their low relative density, can be treated as test particles. In other words, their dynamics can be described by an equation similar to Eq. (1), except that the collisional operator is linearized such that it does not depend on the photoelectron distribution itself. Therefore, the global kinetic collisional test-particle model will provide a sufficient description for the photoelectrons, if the background that consists of the thermal electrons and the ambipolar electric field is determined self-consistently. (Because photoelectron collisions with the ions do not affect the results significantly, but increase the calculation time by a large amount, we neglect them.)

In principle, each ion species should be described by an equation similar to Eq. (1), in which the Coulomb collisional operator depends on the ion distribution function itself because in contrast to photoelectrons, collisions among ions of the same species may no longer be neglected. We circumvent this intrinsic non-linearity with an iterative linearization scheme (Tam et al., 1995a), which will enable us to take such collisions into account. In this scheme, the Coulomb collisions are described by the linearized Fokker-Planck operator. The collisional rates based on this operator are velocitydependent. Thus, the scheme enables us to describe the anisotropic kinetic effects that arise from Coulomb collisions. This iteration scheme, when it converges, takes into account the non-linear dependence of the collisional operator on the distribution function.

\subsection{Advantages of the model}

This self-consistent hybrid technique retains the expediency of fluid theory while in effect extending its applicability by the inclusion of kinetic descriptions. One of the advantages of the model is clearly its ability to incorporate the global kinetic collisional physics of photoelectrons into a self-consistent polar wind description. In fact, this is the first polar wind model to achieve that. As fluid calculations alone fail to describe the nonthermal electron features observed in the polar wind, the technique used in this model not only provides a kinetic description of these features, but also takes into account their impact on the polar wind.

Another difficulty that the self-consistent hybrid model has circumvented is the treatment of the transonic region, which introduces singularities into most moment-based calculations, as discussed in Sect. 1.2. In the polar wind, the flows of both $\mathrm{H}^{+}$and $\mathrm{O}^{+}$are observed to be supersonic at high altitudes. Thus, there must be a transonic region where the nature of the flows changes from subsonic to supersonic. As discussed in Sect. 1.2, most moment-based models have difficulties describing transonic flows because of the singularities intrinsic to the calculations, specifically, those at the sonic points. In the self-consistent hybrid model, however, the dynamics of the ion species are described based on kinetic calculations. Due to the kinetic treatment of the ions, the difficulty arising from the transonic region of the ions is not an issue in this model. Indeed, the model is the first self-consistent global polar wind model to obtain a continuous steady-state solution that varies from a subsonic collisional regime at low altitudes to a supersonic collisionless regime at high altitudes (Tam et al., 1995a).

The model also successfully deals with problems usually encountered by other simulation techniques. For example, simulating the Coulomb collisions among ions of the same species is not trivial, and is usually an obstacle to a self-consistent approach; this model takes into account such "self-collisions" by means of an iterative scheme. The relatively short time scales of thermal electrons, as compared to those of the ions, would slow down the simulation process considerably. In this model, the thermal electrons are described by a fluid approach, and are present in the simulations only as a steady-state background. This technique, by its fluid treatment of the thermal electrons, can evolve using relatively large time steps consistent with the ion and suprathermal electron collisional time scales, and is not constrained by the much shorter thermal electron collisional time scale.

\section{Application to the photoelectron-driven polar wind}

\subsection{Theory, data closure}

The self-consistent hybrid model was developed for the polar wind along open field lines driven by the photoelectron flux, a scenario that corresponds to the daytime polar conditions. Application of the model to the sunlit polar region has generated results (Tam et al., 1995a) that were qualitatively consistent with various observed polar wind features (see Table 2) (Abe et al., 1993a; Yau et al., 1995). This study aims to provide a solution that is more quantitatively similar to the observations at Akebono satellite altitudes. Such an admissible polar wind solution is obtained by appropriately varying the relevant initial and boundary conditions in the ionosphere.

The local electric field in this solution has converged to within $2 \%$ throughout the simulation range 
Table 2. Dayside polar wind observations by the Akebono satellite. Ion measurements were made at $5000-9000 \mathrm{~km}$ altitude (Abe et al., 1993a).

Electron observations were made at about $1700 \mathrm{~km}$ altitude (Yau et al., 1995)

\begin{tabular}{ll}
\hline Qualitative features & Quantitative estimates \\
\hline $\mathrm{O}^{+}$dominance over $\mathrm{H}^{+}$ions & $\mathrm{O}^{+}$density: lower limit $\sim 10^{8} \mathrm{~m}^{-3}$ \\
Monotonically increasing ion outflow velocities & $\mathrm{H}^{+}$density: lower limit $\sim 10^{7} \mathrm{~m}^{-3}$ \\
$\begin{array}{l}\text { Supersonic flows for both } \mathrm{H}^{+} \text {and } \mathrm{O}^{+} \\
\text {Anisotropy between upwardly and }\end{array}$ & Ion temperatures : upper limit $\approx 10^{4} \mathrm{~K}$ \\
downwardly moving electron populations & $\mathrm{H}^{+}$outflow velocity about $12-13 \mathrm{~km} / \mathrm{s}$ \\
Upwardly directed total electron heat flux & $\mathrm{O}^{+}$outflow velocity about $6-7 \mathrm{~km} / \mathrm{s}$ \\
\hline
\end{tabular}

(500 km $-2 R_{\mathrm{E}}$, where $R_{\mathrm{E}}$ is the radius of the earth). In this calculation, initial distributions for the kinetic part of the model are applied at the lower boundary, and are associated with the upper half of the Maxwellian distribution. The ions are initially distributed with the Maxwellian energy spread $\left(T_{*}\right)$ being $0.3 \mathrm{eV}$ for $\mathrm{H}^{+}$and $0.6 \mathrm{eV}$ for $\mathrm{O}^{+}$, while the photoelectrons are of $21.6 \mathrm{eV}$ energy spread, with energy ranging from 2 to $62 \mathrm{eV}$ (based on the photoelectron spectra measured by Lee et al. (1980)). Note that we use "energy spread" instead of the more popular term "temperature" to describe the initial distribution functions, because as will be discussed below, such "temperatures" will be different from their corresponding values in the final result. For the fluid component of the model, boundary conditions are also imposed at the lower end: the $\mathrm{H}^{+}$and $\mathrm{O}^{+}$densities are $2 \times 10^{9}$ and $5 \times 10^{10} \mathrm{~m}^{-3}$ respectively; a photoelectron to thermal electron density ratio of $5 \times 10^{-4}$ is assumed, and the thermal electron temperature is taken to be $3000 \mathrm{~K}$.

The self-consistent electric potential profile for this set of initial and boundary conditions is shown in Fig. 1. A potential drop of about $5 \mathrm{~V}$ is obtained across our simulation range. Note that this potential drop is smaller than that in Tam et al. $(1995 \mathrm{a})(\sim 12 \mathrm{~V})$, but is still considerably larger than most classical polar wind models predicted. This relatively smaller potential drop compared with our previous results (Tam et al., 1995a) is mainly due to a smaller suprathermal to thermal electron density ratio at the boundary. The effect of this boundary density ratio on the polar wind solution will be discussed in detail in Sect. 4.2.

The density profiles for all the species in our calculation are shown in Fig. 2. First of all, the

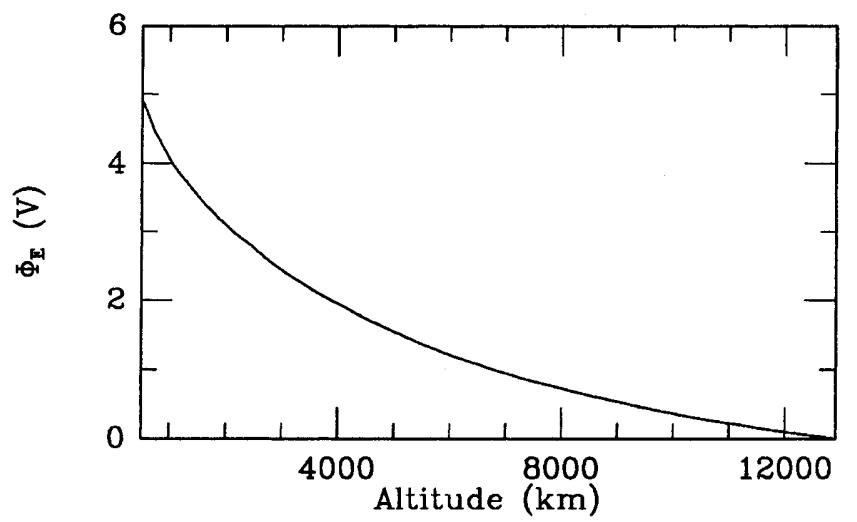

Fig. 1. Electric potential profile calculated by the self-consistent hybrid model. The magnitude of the potential drop is comparable to value deduced from observations photoelectron density $n_{s}$ is much smaller than the thermal electron density. Our test-particle approach for photoelectrons is therefore justified. The results also show that $\mathrm{O}^{+}$, being driven by the self-consistent ambipolar field, maintains its dominance over $\mathrm{H}^{+}$even at high altitudes, in agreement with the Akebono observations. From 5000 to $9000 \mathrm{~km}$, the $\mathrm{O}^{+}$and $\mathrm{H}^{+}$ densities are to the orders of $10^{9}$ and a few times $10^{7} \mathrm{~m}^{-3}$, consistent with the respective lower limit estimates, $10^{8}$ and $10^{7} \mathrm{~m}^{-3}$, obtained from the Akebono measurements (Abe et al., 1993a). Because of the quasineutrality condition, the two major particle species, $\mathrm{O}^{+}$ and thermal electron, have very comparable densities.

Figure 3 shows the outflow velocities of the ions. Note that in general the outflow velocities for both ion species increase with altitude. Not only are these ion velocity profiles consistent with the Akebono observations qualitatively, but, moreover, their high-altitude $(5000-9000 \mathrm{~km})$ values of about $15 \mathrm{~km} / \mathrm{s}$ for the $\mathrm{H}^{+}$, and $4 \mathrm{~km} / \mathrm{s}$ for the $\mathrm{O}^{+}$are also within the measurement limits (Abe et al., 1993a). Of course, when there are special magnetospheric/ionospheric conditions other than the photoelectrons affecting the plasma flow in the polar cap, these results may be altered (Chandler,1995). However, such situations are beyond the scope of this model, in its present form.

The ion parallel and perpendicular temperatures obtained from the kinetic calculations are shown in Fig. 4. The order of temperatures between 5000 and $9000 \mathrm{~km}$ altitude are no higher than $10^{4} \mathrm{~K}$, which is the upper limit estimated from the Akebono measurements (Abe et al., 1993a). Note that these temperatures at the lower boundary may be different from their respective $T_{*}$ values, which characterize the energy spread of the

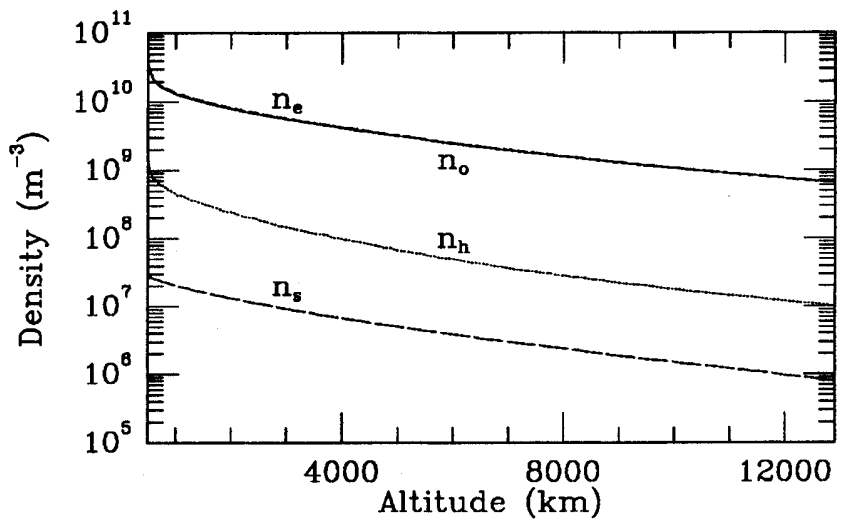

Fig. 2. Calculated density profiles for the $\mathrm{O}^{+}\left(n_{o}\right), \mathrm{H}^{+}\left(n_{h}\right)$, thermal electrons $\left(n_{e}\right)$, and photoelectrons $\left(n_{s}\right)$ 


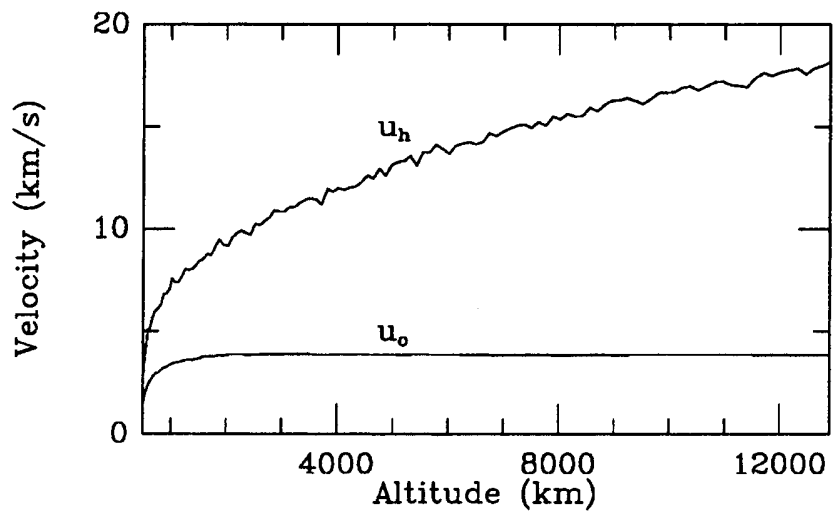

Fig. 3. Profiles of calculated ion outflow velocities

initial distributions. In the calculations, the initial distributions only consist of upgoing ions. These ions may be reflected in the simulations due to Coulomb collisions and the gravitational force. Because the ion temperatures shown in Fig. 4 correspond to the overall ion distributions which comprise both the upgoing and reflected populations, their values at the lower boundary are generally not the same as the initial conditions $T_{*}$.

The Mach number, defined for each ion species $j$ as:

$\mathscr{M}_{j}=\sqrt{\frac{m_{j} u_{j}^{2}}{2 T_{j}}}$

increases in general with altitude. In the case of the $\mathrm{H}^{+}$, the increase is mainly due to the upward gradient of the outflow velocity; while in the case of the $\mathrm{O}^{+}$, the downward gradient of the temperatures is primarily responsible for the Mach number increase (see Figs. 3 and 4). From Fig. 5, it is clear that the continuous solution spans the subsonic and supersonic regimes of both ion species. In particular, both ion species attain supersonic flows at high altitudes, in agreement with the Akebono observations (Abe et al., 1993a).

Note that in our results, the $\mathrm{O}^{+}$outflow becomes supersonic at about $600 \mathrm{~km}$ altitude. However, such a theoretical prediction may not be precise. As mentioned, the increase in the $\mathrm{O}^{+}$Mach number is mainly due to the downward temperature gradient of the species. The $\mathrm{O}^{+}$

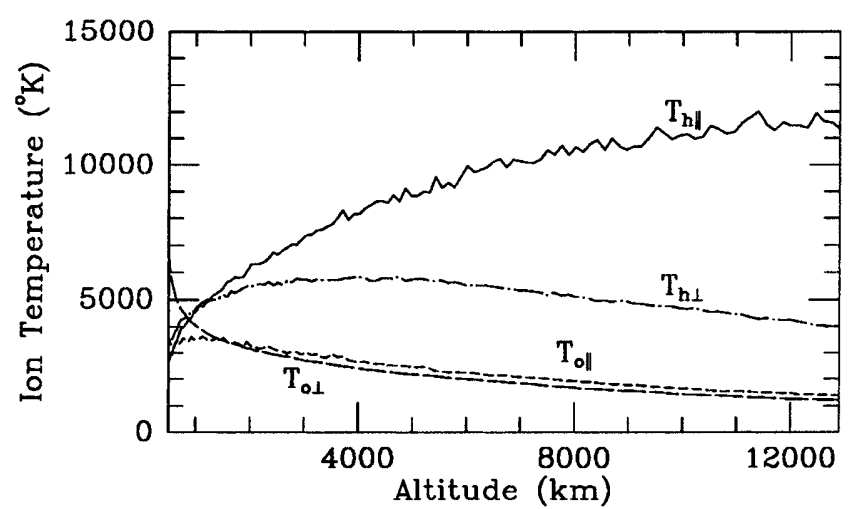

Fig. 4. Profiles of calculated ion temperatures temperatures in our results (see Fig. 4), however, are well below the upper limit estimated from the Akebono data, suggesting that the $\mathrm{O}^{+}$Mach number could be lower than our results predict. If that is indeed the case, the subsonic-to-supersonic transition would occur at a higher altitude. Because the Akebono satellite measurements have not provided a precise polar wind ion temperature at low altitudes, it is difficult for us to give a definite prediction for the location of such a transition.

In order to compare our results with Akebono's electron measurements (Yau et al., 1995), we follow the procedure of Akebono's instrument, and distinguish between electrons according to the sign of their velocity. We combine all the electrons (thermal and photoelectrons) in the calculation into a single population. The thermal electrons in this total population are assumed to be in a drifting Maxwellian distribution whose density, velocity and temperature are determined as explained in Sect. 3. The resulting Maxwellian and the kinetic results for the photoelectrons combine to give the total distribution of the electron population. This total distribution is then divided into two components: $f_{e, \text { up }}$ and $f_{e, \text { down }}$, corresponding to upwardly and downwardly moving electrons, respectively. The parallel temperature profiles obtained from these components are shown in Fig. 6. The solution reveals a temperature anisotropy between the upwardly and downwardly moving electrons, i.e. $T_{e, \text { up }}>T_{e, \text { down }}$ (the $\|$ subscript is omitted for simplicity). In the calculation, this anisotropy is entirely due to the photoelectrons. Because such a temperature anisotropy was observed in the dayside polar wind (Yau et al., 1995) but seems absent on the nightside (Abe et al., 1996), the role of photoelectrons in the model is consistent with the observed polar wind scenario. Despite such a temperature anisotropy introduced by the photoelectrons, the overall temperature profile still seems to be dominated by the thermal electrons. This justifies the use of the thermal electron temperature in the collisional operator of the kinetic calculations, and thus the test-particle approach for photoelectrons.

However, if we consider the next higher order of velocity moment, namely the heat flux, the contribution by the photoelectrons is significant. To show that, we shall compare the heat fluxes carried by the thermal and

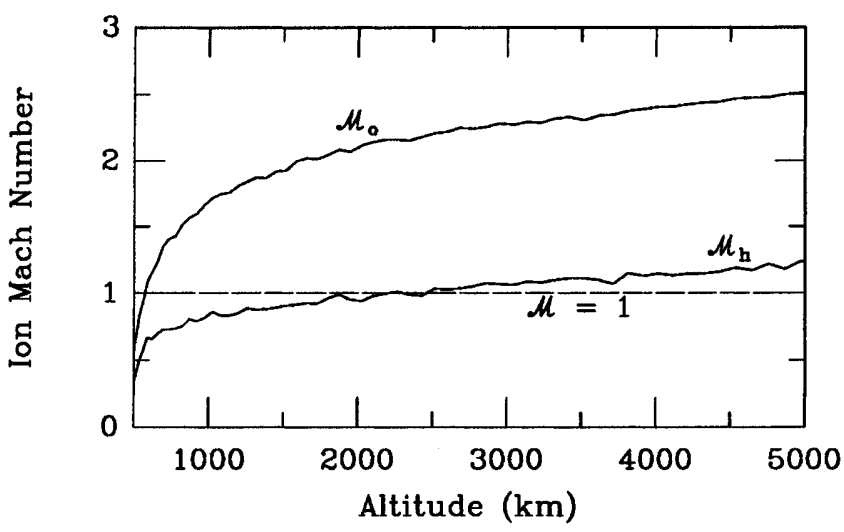

Fig. 5. Ion Mach numbers 


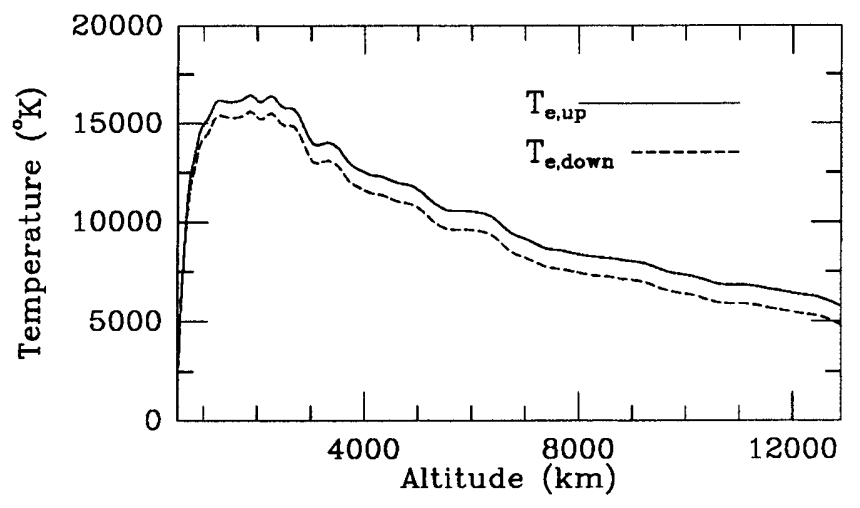

Fig. 6. Parallel temperatures for upwardly and downwardly moving electrons, the $\|$ subscript has been omitted for simplicity

photoelectrons in the total electron distribution. Such heat flux moment should be taken about the mean velocity of the total electron distribution, $u_{e, \text { total }}$. Note that the thermal electrons, though assumed to be distributed in a drifting Maxwellian, have a finite heat flux contribution. This thermal electron heat flux arises from the finite difference between $u_{e, \text { total }}$ and the drift velocity of the Maxwellian itself $\left(u_{e}\right)$. Because the heat flux is proportional to the number density, one would expect that the major contribution to this heat flux comes from the relatively dense portion of the Maxwellian, i.e. the bulk of the thermal electron distribution. In reality, of course, the thermal electron distribution may deviate slightly from a drifting Maxwellian, giving
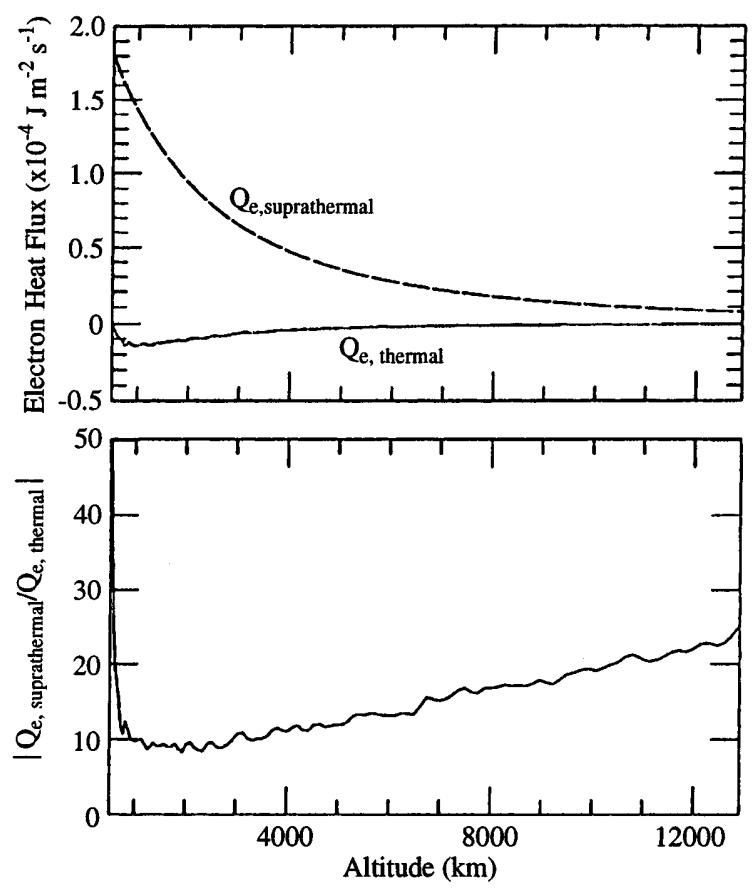

Fig. 7. Top panel: heat fluxes carried by the thermal electrons $\left(Q_{e, \text { thermal }}\right)$ and photoelectrons $\left(Q_{e, \text { suprathermal }}\right)$. Note that the heat flux contribution by the thermal electrons is in the downward direction (negative sign) while that by the photoelectrons is upwardly directed (positive sign). Bottom panel: the ratio of the magnitudes of the two heat fluxes rise to an additional contribution to the heat flux. However, such a heat flux would mainly come from the tail portion of the distribution. We found that this contribution is negligibly small compared with the heat flux carried by the drifting Maxwellian due to the shift in the mean velocity. By comparing the heat flux contribution due to the thermal electron Maxwellian with that carried by the photoelectrons, we find that the heat fluxes carried by the two electron components are in opposite directions, downward for the thermal but upward for the suprathermal, as shown in the top panel of Fig. 7. We also find that the photoelectron heat flux is much larger in magnitude than its thermal counterpart, as shown in the bottom panel of Fig. 7. The two heat flux components combine to give the total electron heat flux, which is shown in Fig. 8 and whose direction is dictated by the photoelectron contribution, i.e. upward. The upwardly directed total electron heat flux is consistent with the data from Yau et al. (1995) and the calculated results by Tam et al. (1995a, b). The results here further agree with Tam et al. (1995b) regarding the directions and the relative magnitudes of the heat fluxes carried by the two electron components. These results, however, might seem different from the observations by Yau et al. (1995), who concluded that both the thermal and photoelectron heat fluxes are upwardly directed, and that the thermal population dominates the overall contribution. The discrepancies between the observations and our results can be explained by the different cutoff energies for the photoelectrons: Yau et al. (1995) only took into account photoelectrons of $>10 \mathrm{eV}$ while recognizing electrons with energy below that as thermal; this study, however, uses a considerably lower energy cutoff $(2 \mathrm{eV})$ for the photoelectron population, which is based on conditions prevailing at the simulation's ionospheric lower boundary. Our results would agree with the observations considering that photoelectrons with energy between 2 and $10 \mathrm{eV}$ make a significant heat flux contribution in the upward direction, even though a fraction of the less energetic electrons in this range will thermalize. (These thermalized photoelectrons are treated kinetically in our model although, strictly speaking, they should be considered as part of the thermal electron background.)

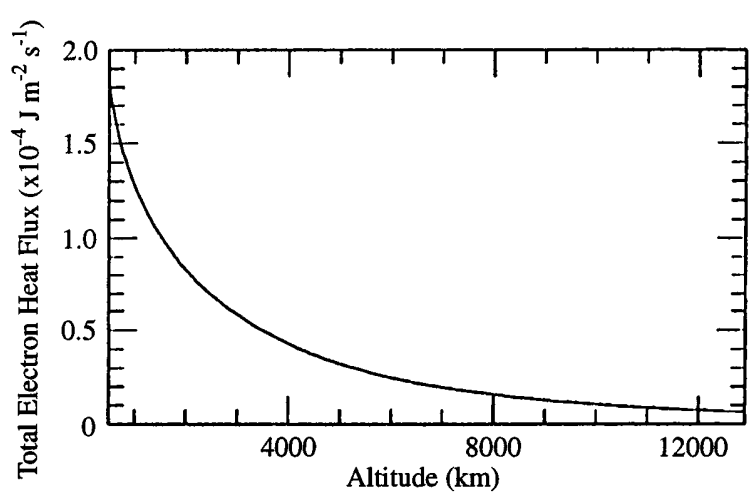

Fig. 8. Total heat flux profile for the total electron population 


\subsection{Day-night transition}

The polar wind results presented in the previous section compare well with observations by the DE-1, DE-2 and Akebono satellites (Winningham and Gurgiolo, 1982; Abe et al., 1993a, b; Yau et al., 1995). These polarorbiting satellites have measured or estimated various polar wind quantities, e.g. the ion and electron outflow characteristics, and the corresponding ambipolar potential difference. The values obtained from the satellite data for these quantities should correspond to those under certain polar wind conditions. However, these observed values may not be representative enough for the polar wind in general because of the variability of the polar wind settings.

Indeed, the dynamics of the polar wind depends on a number of conditions. For example, the correlation between the $\mathrm{H}^{+}$outflow velocity and the amount of magnetic activity, i.e. the $K_{p}$ index, calculated by Abe et al. (1993b) from the Akebono data, seems to indicate an increase of the velocity gradient with the $K_{p}$ index on a time scale of several days. For $K_{p}$ index of shorter time scales, however, no conclusion has been drawn because of insufficient statistical data.

The polar wind dynamics, evidently, also depends on the magnetic local time, as implied by the day-night asymmetries that have been observed by the Akebono satellite (Abe et al., 1993a, 1996; Yau et al., 1995), and discussed in Sect. 1.1. The magnetic local time is related to the solar zenith angle. Indeed, Winningham and Gurgiolo (1982) have observed that the field-aligned electric potential difference of the polar wind varied as the solar zenith angle. Because the solar zenith angle is related to the photoionization rate, which directly contributes to the local ionospheric photoelectron density (Jasperse, 1981), we expect that variation of this density may be closely related to the day-night transition of the polar wind, despite the relatively small photoelectron population compared with the thermal electrons. In this section, we shall examine the day-night transition of the polar wind by studying the effects of the ionospheric photoelectron density.

We should point out that the polar wind dynamics is primarily dictated by the environment in the source region, the ionosphere. Factors such as geomagnetic activity (i.e. the geomagnetic field) and the magnetic local time (i.e. the photoionization rate) prescribe the ionospheric particle distributions, which evolve, giving rise to particle distributions at higher altitudes as the plasma flows upward along the geomagnetic field line. A variation in the ionospheric distributions, therefore, not only reflects a different source environment, but also should lead to different polar outflow dynamics.

Unfortunately, particle distributions possess infinite degrees of freedom (impossible to be represented by a finite number of parameters); to study how the polar wind dynamics varies with the ionospheric distributions, without making further assumptions, is not feasible. We therefore assign the ionospheric particle distributions to a certain form, characterized by only a few parameters, and study how the polar dynamics varies with the boundary parametric values.

We would like to point out that the polar wind dynamics exhibits a complex inter-relationship between all the effects that are present in the plasma outflow. Therefore, it may not be easy to attribute certain changes in a polar wind quantity to a single physical effect. Indeed, as we will show later, some physical quantities are governed by competing effects in the polar wind. Changes in the polar wind photoelectron density, for example, may enhance or diminish these competing effects to different extents, leading to the relative importance of one effect over the other. For this reason, it is necessary to investigate the overall impact of a single factor on the polar wind dynamics based on a selfconsistent model, such as the one used in this study.

To examine the polar wind day-night transition, we should consider the response by the ionospheric photoelectrons. The ionospheric photoelectron distribution is governed by the photoionization spectrum: the amplitude of the spectrum affects the photoelectron density, and its shape determines the shape of the photoelectron distribution. The amplitude of the photoionization spectrum depends on the photoionization rates, which vary with the solar zenith angle, and thus also with the magnetic local time. It is therefore necessary to consider the variation of the photoelectron density in studying the day-night transition of the polar wind. The shape of the photoionization spectrum, on the other hand, varies with the relative abundance of all the molecules and ions in the ionosphere. As the relative molecule and ion abundance is beyond the scope of this study, we shall use an invariant shape for the initial photoelectron distribution throughout this work. In this study, we generate polar wind solutions with different boundary conditions for the relative photoelectron density while keeping other boundary conditions the same. Specifically, the following photoelectron to thermal electron density ratios $n_{s} / n_{e}$ are used at the lower boundary for different cases: (a) 0 ; (b) $5.0 \times 10^{-4}$; (c) $1.0 \times 10^{-3}$; (d) $1.5 \times 10^{-3}$. Cases (a) through (d) thus reflect a transition from night-time to daytime conditions, with a decrease in the solar zenith angle. The parameters used in these cases are summarized in Table 3.

The profiles $n_{s} / n_{e}$ for these cases are shown in Fig. 9. Notice that in case (a), the density ratio is identically zero because of the absence of photoelectrons. In other cases, the photoelectron densities remain small compared with those of the thermal electrons throughout the simulation range. The test-particle approach for photoelectrons is thus justified for these solutions. Figure 10 shows that the relative photoelectron density can significantly affect the self-consistent electric potential difference, in that a higher relative photoelectron density leads to a larger potential difference. Because the photoelectrons are suprathermal particles, they will skew the electron distribution, giving rise to a higher electron energy flux, thereby increasing the ambipolar potential difference. The increase in this potential difference corresponds to a larger local ambipolar electric field at all altitudes, as shown in Fig. 11. 
Table 3. Boundary conditions for the four cases with different relative photoelectron density. Other parameters are common to these cases: $n_{o}\left(\mathrm{O}^{+}\right.$density), $n_{h}\left(\mathrm{H}^{+}\right.$density), $T_{o^{*}}, T_{h^{*}}, T_{s^{*}}$, (energy spread of the initial $\mathrm{O}^{+}, \mathrm{H}^{+}$, and photoelectron distribution), and $T_{e}$ (thermal electron temperature)

\begin{tabular}{lllll}
\hline Case & (a) & (b) & (c) & (d) \\
$n_{s} / n_{e}$ & 0 & $5 \times 10^{-4}$ & $1 \times 10^{-3}$ & $1.5 \times 10^{-3}$ \\
\hline
\end{tabular}

\section{Common}

parameters

$n_{o}\left(\mathrm{~m}^{-3}\right) \quad 4.0 \times 10^{10}$

$n_{h}\left(\mathrm{~m}^{-3}\right) \quad 1.5 \times 10^{9}$

$T_{o^{*}}(\mathrm{eV}) \quad 2.2$

$T_{h^{*}}(\mathrm{eV}) \quad 1.0$

$T_{s^{*}}(\mathrm{eV}) \quad 21.6$

$T_{e}(\mathrm{~K}) \quad 3000$

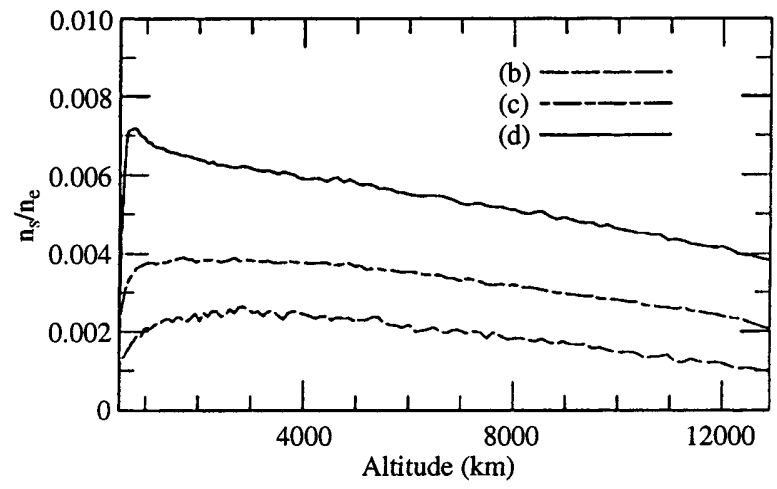

Fig. 9. Profiles of relative photoelectron density. The labels correspond to the cases listed in Table 3

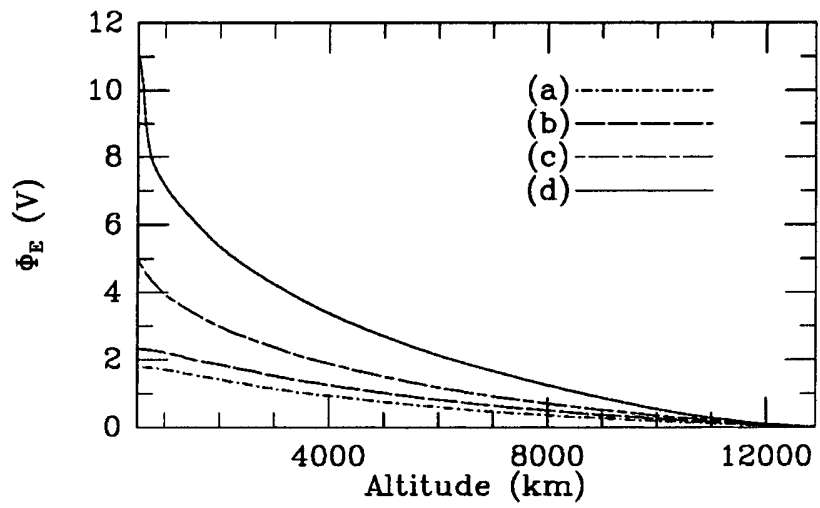

Fig. 10. Profiles of the self-consistent ambipolar electric potential. The labels correspond to the cases listed in Table 3

A higher relative photoelectron density also leads to a higher electron temperature in the low-altitude collisional regime, as Fig. 12 indicates. The upward temperature gradient is related to collisional heating of the thermal electron population. In case (a) where photoelectrons are absent, thermal electrons are heated through their collisions with the ions. When photoelectrons are present, the heating becomes more important as energy is transferred to the thermal electrons also through collisions with the photoelectrons. As the

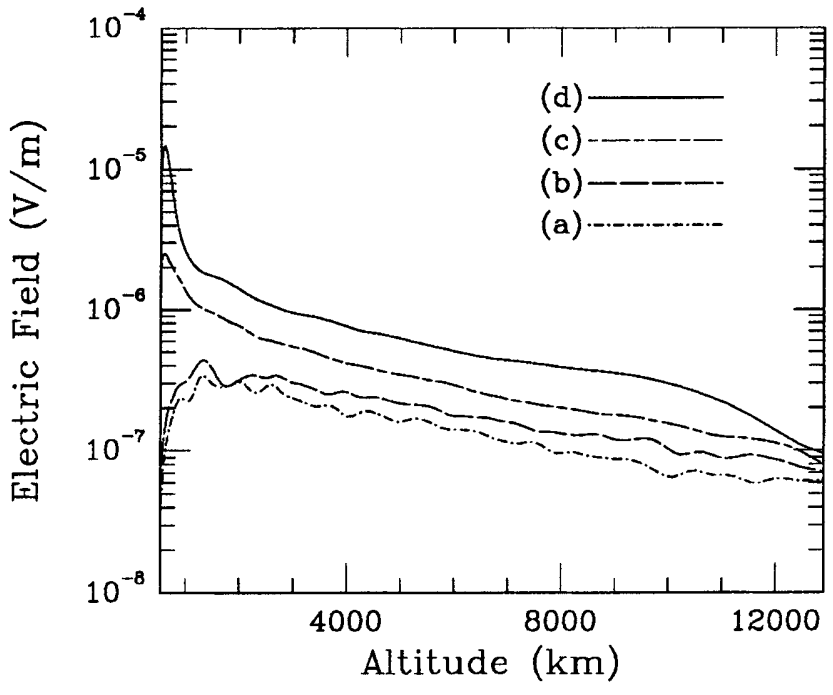

Fig. 11. Profiles of the self-consistent ambipolar electric field. Note that the local electric field increases at all altitudes with the addition of photoelectrons. The labels correspond to the cases listed in Table 3

relative photoelectron density increases, the source of energy provided by the suprathermal population becomes larger, and collisional heating leads to a steeper electron temperature gradient. Because every effect in the polar wind is related due to self-consistency of the dynamics, one may also associate this electron temperature with the electric potential difference, where the two are related in Eqs. (5) and (6) in terms of a balance between competing effects, thermal pressure pushing the electrons outward and the electric field pulling them back.

The impact of the relative photoelectron density on the total electron heat flux, $Q_{e, t o t a l}$, (i.e. the heat flux carried by the combined population of thermal and suprathermal electrons) can be seen in Fig. 13. In the model, because the thermal electron population is assumed to be a Maxwellian, $Q_{e \text {,total }}$ is identically zero in case (a), where photoelectrons are absent. Because $Q_{e, \text { total }}$ is directly related to the number density of the population, it is in fact more correlated with the actual photoelectron density $n_{s}$, as shown in Fig. 14, than with the density ratio $n_{s} / n_{e}$. Nevertheless, the density ratio at the boundary can influence the photoelectron density, not only directly, but also in a non-trivial way through the ambipolar field. Let us consider the transition from (a) through (d) as we increase the relative photoelectron density. Because the density ratio $n_{s} / n_{e}$ is small, as we vary its boundary value, the thermal electron density at the boundary only changes by a negligible amount. Hence, a higher relative photoelectron density means more suprathermal electrons contributing to the tail of the total distribution. Because the upwardly directed electron heat flux is a result of the escape of these energetic particles, more photoelectrons in the distribution, in general, lead to a larger $Q_{e \text {.total }}$ (compare cases (a) through (c)). However, a higher relative photoelectron density, as we have seen, also leads to a larger ambipolar electric field. This field provides an energy 

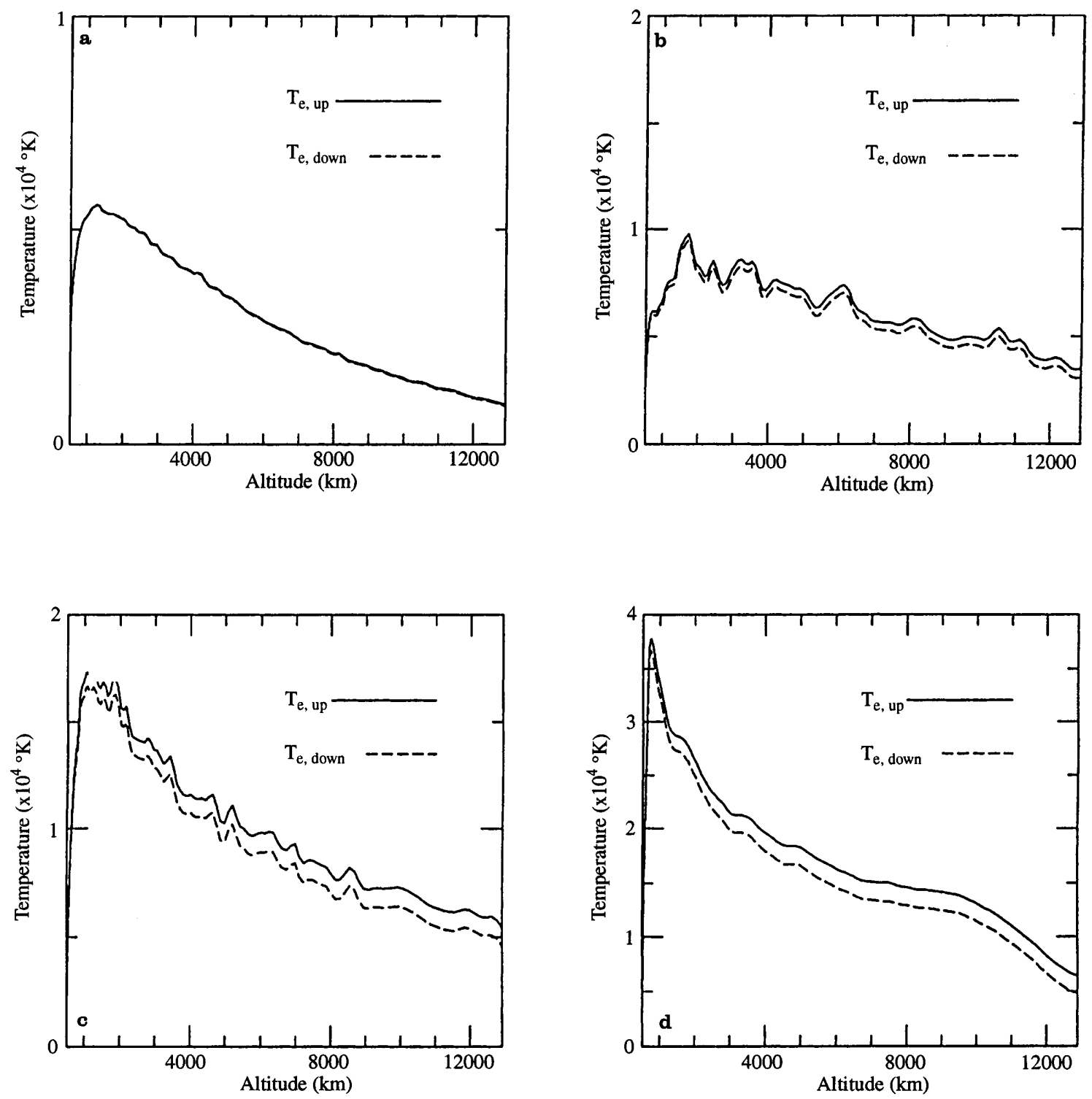

Fig. 12. a-d Parallel temperature profiles for the upwardly and downwardly moving electron populations. The labels correspond to the cases listed in Table 3. Note that the two temperature profiles almost overlap in case (a)

barrier for the escaping electrons, reflecting those with relatively low energies. When the electric field becomes sufficiently large, as a result of the increase in relative photoelectron density, it is able to reflect electrons with energies well in the suprathermal range, significantly weakening the tail portion of the distribution, and lowering $Q_{e, \text { total }}$. The significance of this competing effect due to the electric field is evident, as we compare cases (c) and (d) in terms of photoelectron density and total electron heat flux.

Through its impact on the polar wind ambipolar field, the relative photoelectron density has a significant effect not only on the electron population, but also on the dynamics of the ion outflows. For example, as shown in Table 4, the ion number fluxes generally increase with the relative photoelectron density. This correlation is mainly due to the magnitude of the selfconsistent ambipolar field: the higher the relative pho- toelectron density, the larger the ambipolar field, and consequently, due to acceleration by the field, the greater the number of escaping ions contributing to the number fluxes at high altitudes.

Intuitively, this ion acceleration by the ambipolar field should increase their densities at high altitudes. However, the acceleration itself also creates a competing effect that would lower the ion density, due to conservation of the species. Because mass conservation must hold for every species in the polar wind, the ions, in particular, have to satisfy Eq. (4). In principle, a larger electric field tends to accelerate the ions to higher outflow velocities, thereby reducing their densities to a larger extent. Comparison of the ion density profiles in the four cases, as shown in Fig. 15, provides an indication of the competing effects due to the acceleration by the self-consistent electric field. In general, both the $\mathrm{H}^{+}$and $\mathrm{O}^{+}$densities increase with the relative 


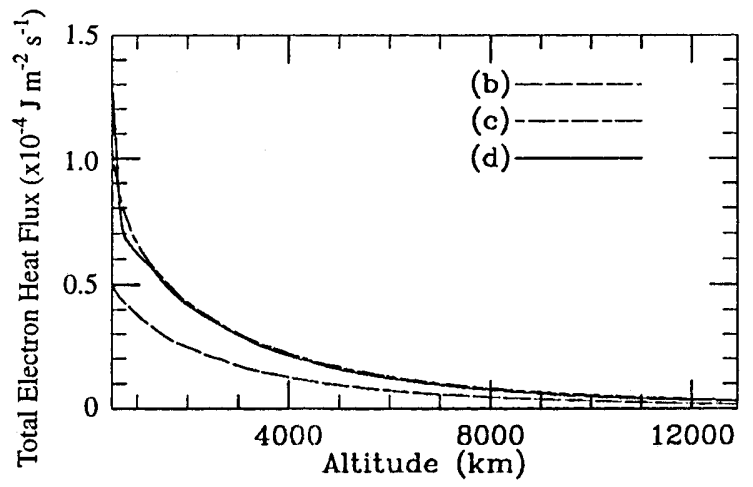

Fig. 13. Total heat flux profiles for the total electron population. The lines for cases (c) and (d) almost overlap in the plot. The labels correspond to the cases listed in Table 3

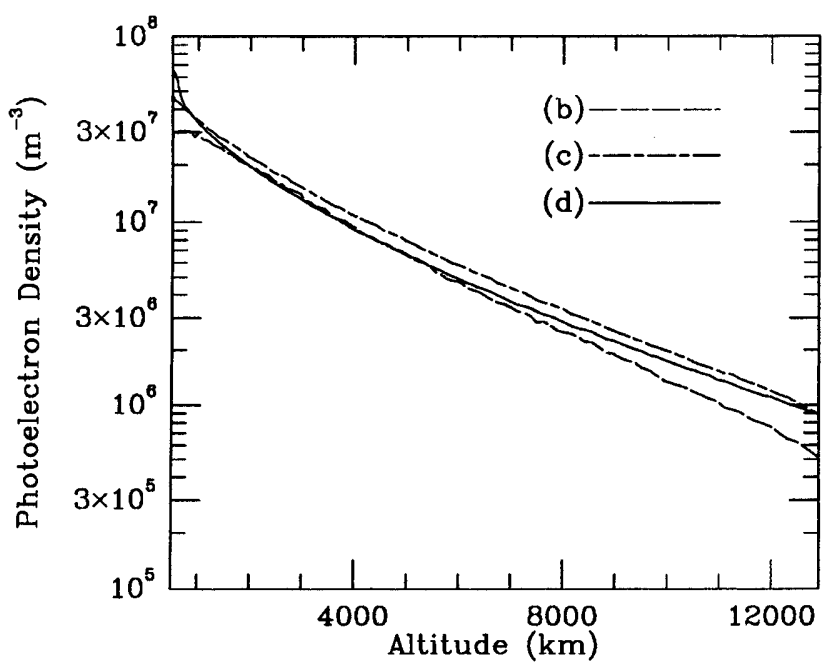

Fig. 14. Profiles of the photoelectron density. The labels correspond to the cases listed in Table 3

photoelectron density, provided that the self-consistent electric field is sufficiently low (compare cases (a) and (b)). However, when the electric field is sufficiently large, the trend is reversed (compare cases (b), (c) and (d)) for the reason discussed already.

Figure 16 shows the velocity profiles of the $\mathrm{O}^{+}$ions in the four cases. The velocity variation with the relative photoelectron density is rather intuitive: the velocity increases with the boundary density ratio $n_{s} / n_{e}$, due to the magnitude of the self-consistent ambipolar field.

Table 4. A list of the density ratio $n_{s} / n_{e}$ at $500 \mathrm{~km}$ altitude, and the ion number fluxes for the four cases, whose boundary conditions are shown in Table 3

\begin{tabular}{llll}
\hline Case & $n_{\mathrm{s}} / n_{\mathrm{e}}$ & \multicolumn{2}{l}{ Number flux at $10000 \mathrm{~km}^{2}\left(\mathrm{~m}^{-2} \mathrm{~s}^{-1}\right)$} \\
\cline { 3 - 4 } & & $\mathrm{H}^{+}$ & $\mathrm{O}^{+}$ \\
\hline (a) & 0 & $9.49 \times 10^{10}$ & $3.25 \times 10^{11}$ \\
(b) & $5 \times 10^{-4}$ & $1.24 \times 10^{11}$ & $1.82 \times 10^{12}$ \\
(c) & $1 \times 10^{-3}$ & $2.35 \times 10^{11}$ & $3.20 \times 10^{12}$ \\
(d) & $1.5 \times 10^{-3}$ & $2.61 \times 10^{11}$ & $3.19 \times 10^{12}$ \\
\hline
\end{tabular}

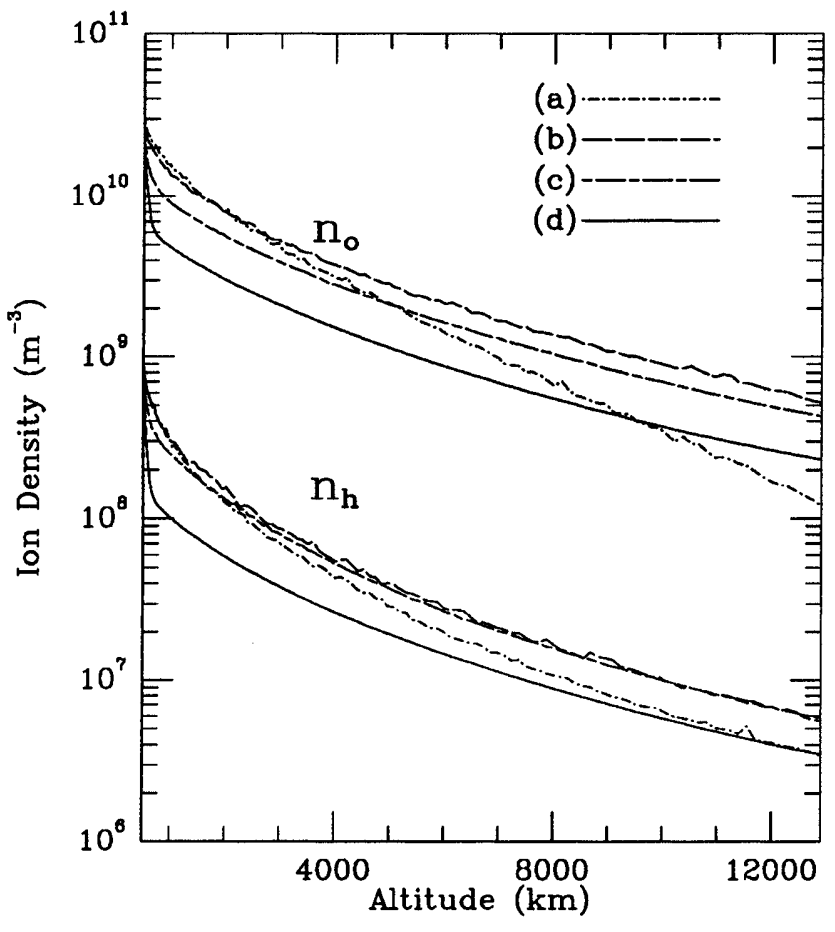

Fig. 15. Profiles of the $\mathrm{O}^{+}$and $\mathrm{H}^{+}$densities. The labels correspond to the cases listed in Table 3

This variation is consistent with the observed day-night asymmetry in the polar wind ion outflow velocities (Abe et al., 1993a). It is also interesting to note that the $\mathrm{O}^{+}$ velocity gradient becomes very small at high altitudes in all four cases. In order to understand why, let us consider the acceleration of an $\mathrm{O}^{+}$ion due to the combination of the electric and gravitational fields. Figure 17 shows the acceleration $e E / m_{o}-g$ for all four cases, where the subscript $\|$ in $E_{\|}$is dropped for simplicity hereafter. Note that the $\mathrm{O}^{+}$ions are in fact decelerated by this combined field at all altitudes in both cases (a) and (b), as $e E / m_{o}-g$ is negative throughout our simulation range. Case (d), however, is almost the exact opposite; the $\mathrm{O}^{+}$ions are accelerated upward until they reach $11000 \mathrm{~km}$ altitude. The small velocity

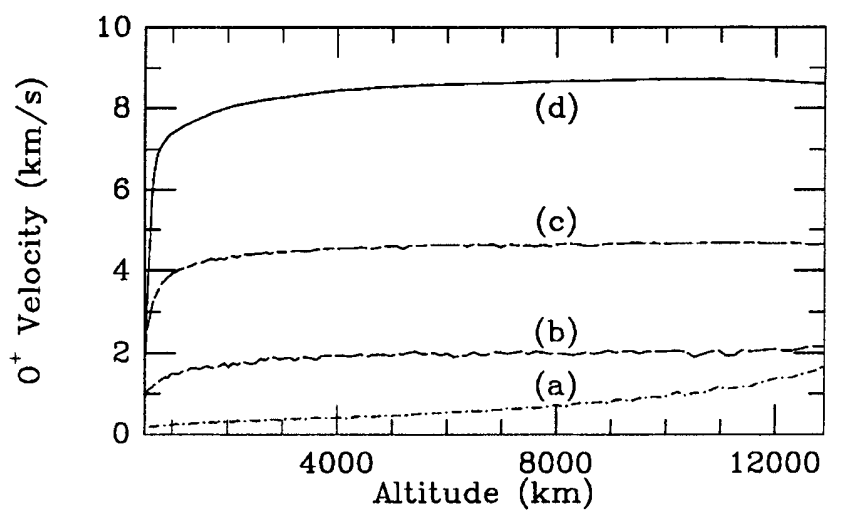

Fig. 16. Profiles of the $\mathrm{O}^{+}$outflow velocity. The labels correspond to the cases listed in Table 3 
gradient around $6000-11000 \mathrm{~km}$ altitude in this case is the result of the small upward acceleration by this combined field. Case (c) is the intermediate case, where the $\mathrm{O}^{+}$ions are accelerated upward by the combined field below $1000 \mathrm{~km}$ altitude, but slightly decelerated above that. Because of the upward acceleration, the $\mathrm{O}^{+}$ velocity increases relatively rapidly with altitude below $1000 \mathrm{~km}$, as compared with the high-altitude situation. Because all four cases feature a trend of increasing $\mathrm{O}^{+}$ velocity with altitudes, the velocity profiles in cases (a) and (b), and at high altitudes in (c), may seem contrary to intuition.

However, this counter-intuitive behavior can be understood from a global, kinetic picture where particle distributions evolve along the field line. To simplify the picture, we shall limit our discussion to only one dimension, namely, the field-aligned direction, and neglect collisions and the inhomogeneity of the magnetic field for the time being. Now let us consider a particle distribution with an upward mean velocity at some altitude $s_{0}$, above which the particle species experiences a net force in the downward direction due to the combined action of the gravitational and electric fields. Because of this downward force, the potential energy for these particles increases with altitude. Due to energy conservation, not all the particles from $s_{0}$ can reach a
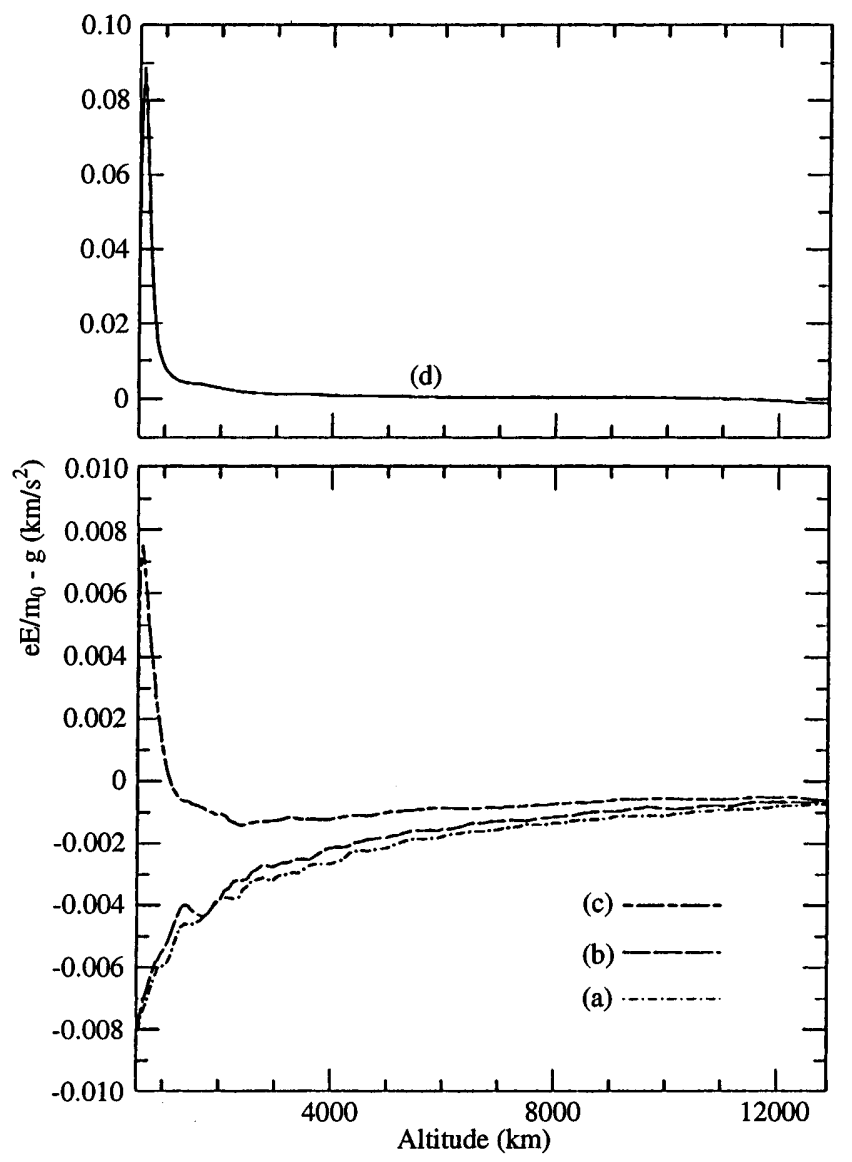

Fig. 17. Profiles of the $\mathrm{O}^{+}$acceleration due to the combination of the electric and gravitational fields. The labels correspond to the cases listed in Table 3 higher altitude $s$. Only those particles with sufficient kinetic energy at $s_{0}$ are able to arrive at altitude $s$. This scenario is illustrated in Fig. 18. In the figure, the shaded region represents the particles at $s_{0}$ that do not reach $s$. Note that the average velocity among the particles in the shaded region would be smaller than the mean velocity among all the particles at $s_{0}$. The absence of these particles at $s$ tends to lead to a higher local mean velocity there. Thus, this global kinetic effect might give rise to an upward velocity gradient. However, one must also take into account the velocity change of the individual particles that exist at both $s_{0}$ and $s$ in determining the direction of the velocity gradient for the species.

Therefore, let us examine this global kinetic effect due to particle reflection in a more quantitative way, based on an alternative reasoning. In the simplified geometry discussed above, the mass conservation equation for any given species is:

$\frac{\partial}{\partial s}(n u)=0$.

Thus, the particle flux is the same at all altitudes. Now suppose the gravitational force on the $\mathrm{O}^{+}$ions is stronger than the electric force everywhere above $s_{0}$. Then the potential energy for the $\mathrm{O}^{+}$ions, defined as
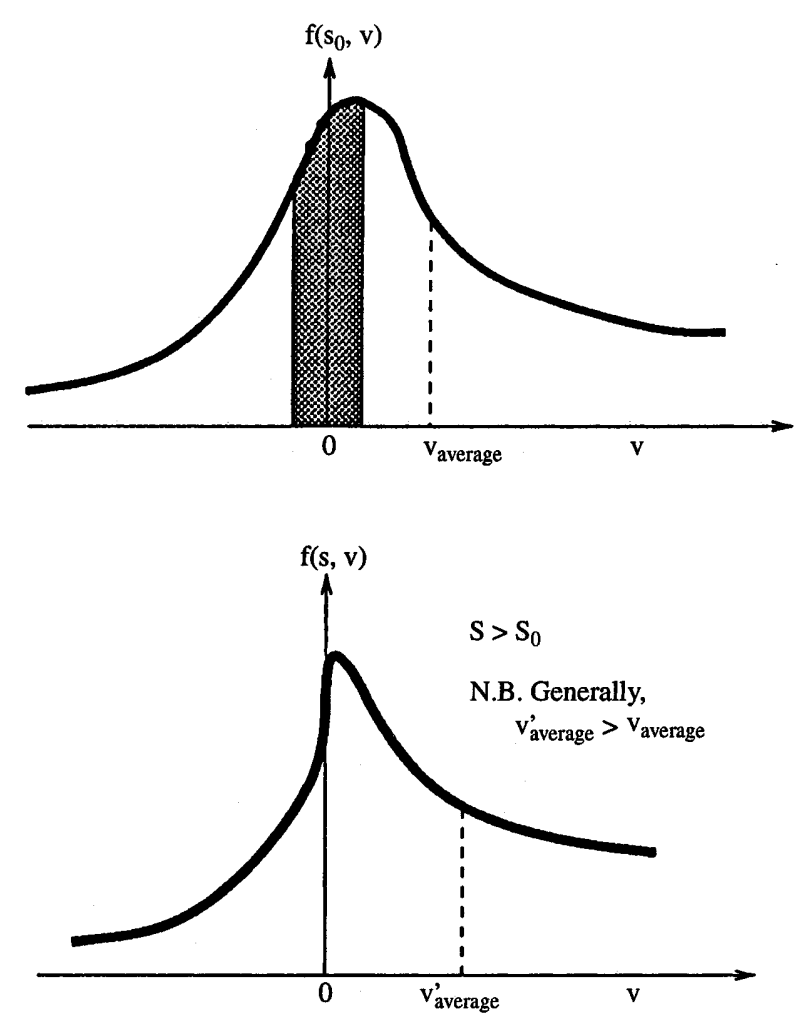

Fig. 18. Illustration of particle distribution evolution under an upward potential energy gradient. The shaded region corresponds to those particles that do not have sufficient kinetic energy to reach $s$. These particles will be reflected between $s_{0}$ and $s$ by the potential barrier, and thus make no contribution to the distribution at $s$. The kinetic effect due to these reflected particles generally leads to a larger average velocity at $s$ than at $s_{0}$ 
$\phi(s)=m\left[\Phi_{G}(s)-\Phi_{G}\left(s_{0}\right)\right]+\mathrm{e}\left[\Phi_{E}(s)-\Phi_{E}\left(s_{0}\right)\right]$

would be positive, and increase with altitude for $s>s_{0}$. (The subscript o for $\mathrm{O}^{+}$has been dropped for simplicity.) Using energy conservation, we can map an ion's velocity $v$ at $s$ back to its velocity $v_{0}$ at $s_{0}$, giving

$v_{0}=\frac{v}{|v|} \sqrt{v^{2}+\frac{2 \phi(s)}{m}}$.

Because $\phi(s)>0$, the energy barrier prevents those $\mathrm{O}^{+}$ ions at $s_{0}$ with speed $\left|v_{0}\right|<\sqrt{2 \phi(s) / m}$ from reaching $s$. In other words, these ions make no contribution to the density and particle flux at $s$. These ions also do not have a net contribution to the particle flux at $s_{0}$ because due to energy conservation, each of them would come down with a velocity equal and opposite to that in its upward leg. However, they contribute to the density at $s_{0}$ every time they reach there. These ions, therefore, may lead to a downward gradient in density. According to Eq. (8), that would correspond to an upward gradient in the local mean velocity, even though the net force is downward throughout the altitudes above $s_{0}$.

To illustrate this discussion, let us assume that the steady-state $\mathrm{O}^{+}$distribution function at $s_{0}$ is a Maxwellian with drift velocity $u_{0}>0$, i.e.

$f\left(s_{0}, v\right)=n_{0} \sqrt{\frac{m}{2 \pi T_{0}}} \exp \left(-\frac{m\left(v-u_{0}\right)^{2}}{2 T_{0}}\right)$,

where $n_{o}$ and $T_{0}$ are respectively the species' density and temperature at $s_{0}$. Using Eq. (10), the distribution function at $s>s_{0}$ is then:

$$
\begin{aligned}
f(s, v)= & \theta(v) n_{0} \sqrt{\frac{m}{2 \pi T_{0}}} \exp \left[-\left(\sqrt{\frac{m v^{2}}{2 T_{0}}+\frac{\phi}{T_{0}}}-\sqrt{\frac{m u_{0}^{2}}{2 T_{0}}}\right)^{2}\right] \\
& +\theta(-v) n_{0} \sqrt{\frac{m}{2 \pi T_{0}}} \\
& \exp \left[-\left(-\sqrt{\frac{m v^{2}}{2 T_{0}}+\frac{\phi}{T_{0}}}-\sqrt{\frac{m u_{0}^{2}}{2 T_{0}}}\right)^{2}\right]
\end{aligned}
$$

where

$\theta(x)= \begin{cases}1 & \text { for } x \geq 0 \\ 0 & \text { for } x<0\end{cases}$

One can show that the density at $s$ is given by the expression:

$n(s)=\frac{n_{0}}{\sqrt{\pi}} \int_{0}^{\infty} \mathrm{d} x 2 \mathrm{e}^{-\left(x^{2}+\Delta+\mathscr{M}_{0}^{2}\right)} \cosh \left(2 \mathscr{M}_{0} \sqrt{x^{2}+\Delta}\right)$,

where $x \equiv \sqrt{m /\left(2 T_{0}\right)} v, \Delta(s) \equiv \phi(s) I_{0}, \quad$ and $\quad \mathscr{M}_{0} \equiv$ $\sqrt{m /\left(2 T_{0}\right)} u_{0}$. From Eq. (13), we can obtain:

$$
\begin{aligned}
\frac{\partial n}{\partial \Delta}= & \frac{n_{0}}{\sqrt{\pi}} \int_{0}^{\infty} \mathrm{d} x 2 \mathrm{e}^{-\left(x^{2}+\Delta+\mathscr{M}_{0}^{2}\right)} \\
& \times\left\{\frac{\mathscr{M}_{0}}{\sqrt{x^{2}+\Delta}} \sinh \left(2 \mathscr{M}_{0} \sqrt{x^{2}+\Delta}\right) .\right. \\
& \left.-\cosh \left(2 \mathscr{M}_{0} \sqrt{x^{2}+\Delta}\right)\right\} .(14)
\end{aligned}
$$

With Eqs. (8), (9), (13), and (14), we can then obtain the value for

$$
\begin{aligned}
\frac{1}{u(s)} \frac{\partial u(s)}{\partial s} & =-\frac{1}{n(s)} \frac{\partial n}{\partial \Delta} \frac{\partial \Delta}{\partial s} \\
& =-\frac{1}{n(s)} \frac{\partial n}{\partial \Delta}\left(\frac{m g-e E}{T_{0}}\right)
\end{aligned}
$$

where we have made use of $g=\partial \Phi_{G} / \partial s$ and $E=-\partial \Phi_{E} / \partial s$. We have evaluated $(1 / n)(\partial n / \partial \Delta)$ with various combinations of $\Delta$ and $\mathscr{M}_{0}$. These values are shown in Table 5. Because $m g-e E$ is positive by the assumption of a downward force on the $\mathrm{O}^{+}$, and $u(s)=n_{0} u_{0} / n(s)>0$, we can see from Table 5 that $\partial u / \partial s$ can be positive or negative depending on the values of the parameters. However, one can conclude that in general, $(1 / u)(\partial u / \partial s)$ is more positive or less negative with a smaller $u_{0}$. That is because with a smaller $u_{0}$, more $\mathrm{O}^{+}$ions will be readily reflected, and thereby more likely to result in a downward density gradient. The magnitude of the velocity scale length, however, is also directly related to the magnitude of the force due to the combined field, as indicated in Eq. (15). If this force is small, the velocity scale length will tend to be large.

The global kinetic effect discussed above appears in cases (a), (b), and (c) of our results, particularly at $3000 \mathrm{~km}$ altitude and above, where the effect of Coulomb collisions is small, and where the combined force due to the electric and gravitational fields is downward (see Fig. 17). In these cases, $\left(m_{o} g-e E\right) / T_{o \|}$ is of the order of $10^{-4} \mathrm{~km}^{-1}$, and $|(1 / n)(\partial n / \partial \Delta)|$ is of the order of unity. According to Eq. (15), the velocity scale lengths for these cases are thousands of kilometers. These long scale lengths at high altitudes are consistent with the results shown in Fig. 16. This verifies that the velocity increase for the $\mathrm{O}^{+}$ions in cases (a), (b), and (c) is due to the kinetic effect that arises from the reflection of the ions.

Figure 19 shows the $\mathrm{H}^{+}$outflow velocity profiles. Like the $\mathrm{O}^{+}$velocity, the $\mathrm{H}^{+}$velocity generally also increases with the boundary density ratio $n_{s} / n_{e}$, except

Table 5. $(1 / n)(\partial n / \partial \Delta)$ for various values of the parameters $\mathscr{M}_{0}$ and $\Delta$. The calculations are based on Eqs. (13) and (14)

\begin{tabular}{llllrrr}
\hline$\Delta$ & $\mathscr{M}_{0}$ & & & & & \\
\cline { 2 - 7 } & 0.25 & 0.5 & 0.75 & \multicolumn{1}{c}{1.0} & \multicolumn{1}{c}{1.25} & \multicolumn{1}{l}{2.0} \\
\hline 0.1 & -0.881 & -0.586 & -0.248 & 0.025 & 0.188 & 0.202 \\
0.5 & -0.885 & -0.624 & -0.350 & -0.127 & 0.031 & 0.177 \\
1.0 & -0.889 & -0.660 & -0.437 & -0.251 & -0.101 & 0.131 \\
3.0 & -0.902 & -0.747 & -0.608 & -0.481 & -0.362 & -0.063 \\
\hline
\end{tabular}


that its variation is somewhat more complicated. As we see from Fig. 19, case (b) generally has a smaller $\mathrm{H}^{+}$ outflow velocity than case (a), contrary to intuition considering that the self-consistent electric field is larger in (b). In fact, this piece of information provides an excellent example demonstrating the complexity of the global kinetic collisional dynamics of the polar wind; the counter-intuitive result is due to the Coulomb collisions between the $\mathrm{H}^{+}$and $\mathrm{O}^{+}$ions.

Note that in our results (and the Akebono observations Abe et al., 1993a), $\mathrm{H}^{+}$is the minority ion species; an $\mathrm{H}^{+}$ion will collide mostly with the $\mathrm{O}^{+}$. Because the $\mathrm{O}^{+}$has a smaller outflow velocity than the $\mathrm{H}^{+}$, the Coulomb interaction between the two species will slow down the latter. The significance of this slowing-down effect on the $\mathrm{H}^{+}$depends on the efficiency of the collisions. One way to describe this efficiency is to compare the local time scales of the collisional process with the local $\mathrm{H}^{+}$transit time

$\tau_{\text {transit }}=L / u_{h}$,

where $L$ is the local scale length. Note that $\tau_{\text {transit }}$ characterizes the time scale that a typical $\mathrm{H}^{+}$ion would stay in the local region. For the present purpose, we are interested in comparing $\tau_{\text {transit }}$ with the collisional time $\tau_{\text {coll }}$ that characterizes $\mathrm{H}^{+}$collisions with the $\mathrm{O}^{+}$, i.e.

$\tau_{\text {coll }}=v_{\text {coll }}^{-1}$,

where $v_{\text {coll }}$ represents the collisional frequencies due to slowing-down, and parallel and perpendicular diffusion. The specific expressions for these collisional frequencies can be found in Book (1989). There are thus three characteristic collisional times: $\tau_{\text {coll,s }}$ for the slowingdown effect, and $\tau_{\text {coll }, \|}$ and $\tau_{\text {coll }, \perp}$ for diffusion. Table 6 shows these characteristic times for the $\mathrm{H}^{+}-\mathrm{O}^{+}$interaction (which is the most significant type of Coulomb collisions for the $\mathrm{H}^{+}$species), and $\tau_{\text {transit }}$ (based on the local $\mathrm{O}^{+}$scale length), and the ratios of these time scales, all at $5000 \mathrm{~km}$ altitude. Note that in particular, the ratio $\tau_{\text {transit }} / \tau_{\text {coll, }}$ indicates the efficiency of the slowing-down effect on the $\mathrm{H}^{+}$, the larger the ratio, the more an $\mathrm{H}^{+}$ion is slowed down by its interaction with the $\mathrm{O}^{+}$. It is clear that this ratio is larger in case (b) than in (a), suggesting that $\mathrm{H}^{+}$ions are subjected to the slowing-down effect to

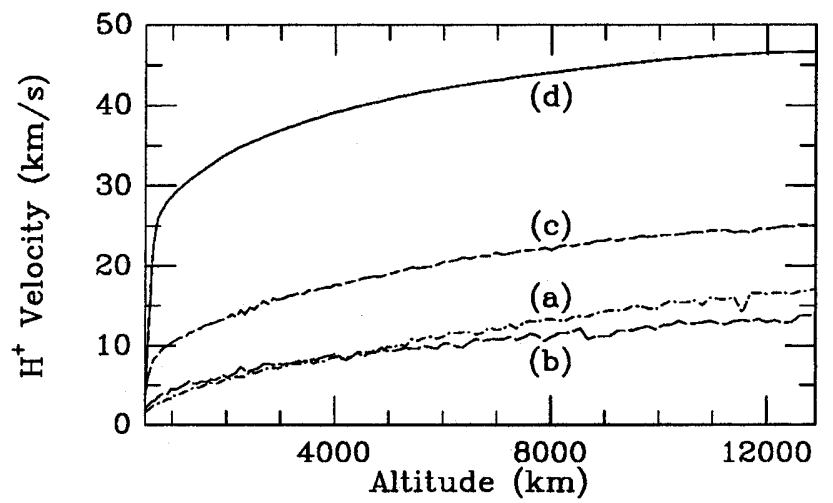

Fig. 19. Profiles of the $\mathrm{H}^{+}$outflow velocity. The labels correspond to the cases listed in Table 3
Table 6. $\mathrm{H}^{+}$time scales at $5000 \mathrm{~km}$ altitude

\begin{tabular}{lllll}
\hline Case & (a) & (b) & (c) & (d) \\
\hline$\tau_{\text {transit }}(\mathrm{s})$ & 256.7 & 208.6 & 200.0 & 91.78 \\
$\tau_{\text {coll,s }}(\mathrm{s})$ & 84.53 & 31.40 & 302.3 & 16605 \\
$\tau_{\text {coll }, \|}(\mathrm{s})$ & 1049 & 381.8 & 12909.9 & $2.020 \times 10^{6}$ \\
$\tau_{\text {coll, } \perp}(\mathrm{s})$ & 93.81 & 34.88 & 325.2 & 7027 \\
$\tau_{\text {transit }} / \tau_{\text {coll, }}$ & 3.037 & 6.643 & 0.662 & 0.014 \\
$\tau_{\text {transit }} / \tau_{\text {coll, }}$ & 0.245 & 0.546 & 0.015 & $4.543 \times 10^{-5}$ \\
$\tau_{\text {transit }} / \tau_{\text {coll, } \perp}$ & 2.736 & 5.981 & 0.615 & 0.013 \\
\hline
\end{tabular}

a larger extent in (b), resulting in an overall smaller outflow velocity. Although we have only shown the time scales at one local point, these ratios, for our comparison purpose, sufficiently characterize the role of collisions in our polar wind solutions. In order to show that this collisional slowing-down effect is indeed comparable to that due to the ambipolar electric field on the $\mathrm{H}^{+}$ ions, we plot the magnitudes of the acceleration/ deceleration by the two effects in Fig. 20 for cases (a) and (b). Note that the altitude range in Fig. 20 begins from $3000 \mathrm{~km}$, which is about the altitude where the $\mathrm{H}^{+}$ velocities for cases (a) and (b) intersect in Fig. 19. Below that altitude, the acceleration/deceleration can be higher by an order of magnitude. Therefore, we exclude the low-altitude range for the clarity of the figure. As Fig. 20 shows, in both cases (a) and (b), the deceleration due to $\mathrm{H}^{+}-\mathrm{O}^{+}$Coulomb collisions is of the same order of magnitude as the acceleration by the ambipolar electric field for the $\mathrm{H}^{+}$ions. The figure also indicates that the collisional slowing-down is more effective in case (b) than in case (a), as the comparison of $\tau_{\text {coll,s }}$ and $\tau_{\text {transit }}$ suggests.

Collisions also play a significant role in shaping the polar wind ion temperature profiles. In particular, collisions lead to velocity diffusion in the distribution of the species, which tends to increase the temperatures as the particles move along the field lines. Because ions in the polar wind are predominantly upstreaming, we would expect diffusion to contribute to a temperature increase with altitude. Figure 21 shows the $\mathrm{H}^{+}$and $\mathrm{O}^{+}$ temperatures in the four polar wind cases. (Because the calculation results have not been smoothed, wiggles are still present in the lines, reflecting the statistical nature of the Monte Carlo technique. These wiggles, however, do not affect the general trend of the results.) The collisional impact is most noticeable when we look at the profiles for the $\mathrm{H}^{+}$parallel temperature $T_{h \|}$. In particular, $T_{h \|}$ is much lower in case (d) than in the other cases. One of the reasons for that is the relatively collisionless nature of the $\mathrm{H}^{+}$outflow, or in other words, the relatively low $\mathrm{H}^{+}$diffusion rates for the case. (The diffusion rates are proportional to the density of the field species. Note that the densities $n_{o}, n_{h}$, and $n_{e} \approx n_{o}+n_{h}$ are generally lower in case (d) than in the other cases.) Of course, the overall velocity diffusion for a given species results from collisions with each particle species, including the species itself whose diffusion is described. To demonstrate that case (d) is indeed the least collisional case for the $\mathrm{H}^{+}$, we will, however, highlight only its collisions with the $\mathrm{O}^{+}$, which is the major ion 


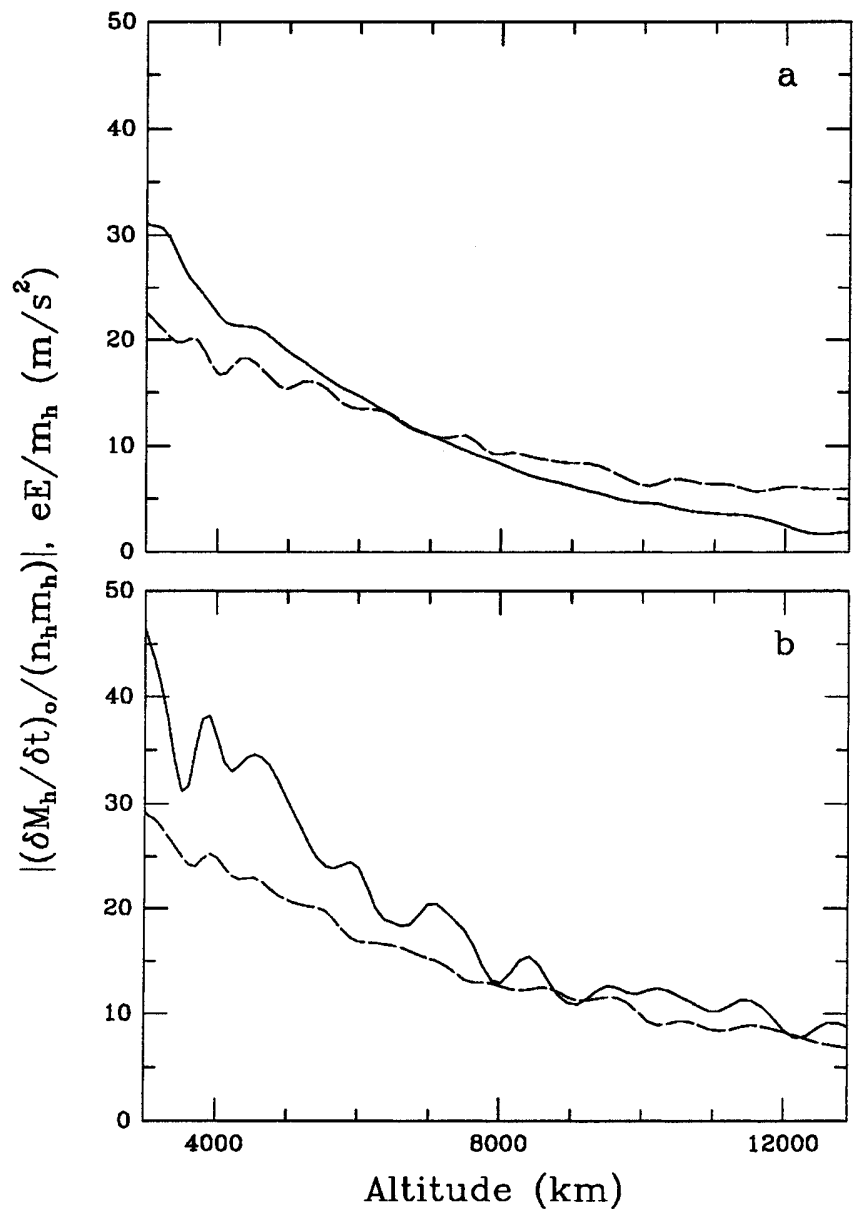

Fig. 20. Comparison of $\mathrm{H}^{+}$deceleration due to $\mathrm{H}^{+}-\mathrm{O}^{+}$Coulomb collisions (solid) with its acceleration due to the ambipolar electric field (dashed). Note that $\left(\delta M_{h} / \delta t\right)_{o} /\left(n_{h} m_{h}\right)$, which is the average deceleration of an $\mathrm{H}^{+}$ion due to $\mathrm{H}^{+}-\mathrm{O}^{+}$collisions, is negative throughout the altitude range, while $e E / m_{h}$ is always positive. The labels $(a)$ and $(b)$ correspond to the cases listed in Table 3

species in both the polar wind observations and our solutions. These $\mathrm{H}^{+}-\mathrm{O}^{+}$interactions, whose collisional times at $5000 \mathrm{~km}$ altitude are shown and compared with the transit times in Table 6, are sufficient to characterize the $\mathrm{H}^{+}$collisions for our present purpose. As the table indicates, $\mathrm{H}^{+}$ions in case (d) have a small ratio of $\tau_{\text {transit }} / \tau_{\text {coll }, \perp}$, and an even much smaller ratio of $\tau_{\text {transit }} / \tau_{\text {coll }, \|}$. (Note that the parallel and perpendicular directions here are relative to the vectorial difference between the velocities of individual $\mathrm{H}^{+}$ions and the outflow velocity of the $\mathrm{O}^{+}$species. Because the outflow velocities of the two ion species are very different, the vectorial difference that we refer to is almost fieldaligned.) Hence, velocity diffusion due to collisions, especially in the field-aligned direction, is rather insignificant in this particular case. In comparison, collisional effects on the $\mathrm{H}^{+}$ions are more important in the other cases, and diffusion may dominate in the complex interplay among other polar wind effects, giving rise to an upward gradient in $T_{h \|}$ at some altitudes. Similarly, the upward gradients in $T_{h \perp}$ at low altitudes are also due to the dominance of diffusion.
In contrast to the diffusion effect which tends to create an upward gradient in the ion temperature, acceleration of the ions by the field-aligned electric field tends to do the opposite, and is largely responsible for the downward gradients of $T_{h \|}$ and $T_{o \|}$ at low altitudes in case (d). This effect arises because of the global steady-state nature of the polar wind. To understand this effect, let us consider particles of the same species but with different velocities flowing out from altitude $s_{1}$ to $s_{2}$ while being accelerated by a field-aligned electric field. For a particle with a high outflow velocity, the time it takes to cover the altitude range is short. Because of the short duration, the momentum it receives from the electric field is small. By the same token, the momentum transfer to a particle of small outflow velocity will be large, although the particle will reach $s_{2}$ at a later time. However, under steady-state considerations, the arrival time is not an issue. An observer of the steady-state flow simply sees that the outflow velocity differential among the particles reduces as they move upward. Reduction of the outflow velocity differential, of course, means a decrease in the parallel temperature of the species. The impact on the temperature due to this velocity-differential momentum transfer depends on the strength of the electric field: the higher the electric field, the more significant its impact. Because the electric field in our polar wind solutions (see Fig. 11) is generally larger in case (d) and at low altitudes, its effect, on the temperature is most noticeable there, where it dominates the diffusion effect, leading to decreasing parallel temperatures for both ion species.

In fact, the ion parallel temperature gradients are strongly related to the size and direction of the forces. This can be demonstrated with $T_{o \|}$ in our results. If we take the first-order moment, namely the momentum, of Eq. (1) for the $\mathrm{O}^{+}$ions, dropping the time-dependence term and using Eq. (4), we can arrive at the following expression that involves the pressure gradient:

$$
\begin{aligned}
\frac{1}{n_{o}} \frac{\partial}{\partial s}\left(n_{o} T_{o \|}\right)= & -m_{o} u_{o} \frac{\partial u_{o}}{\partial s}+\frac{B^{\prime}}{B}\left(T_{o \|}-T_{o \perp}\right) \\
& +\left(e E-m_{o} g\right)+\frac{1}{n_{o}} \frac{\delta M_{o}}{\delta t}
\end{aligned}
$$

where $\delta M_{o} / \delta t$ is the rate of momentum transfer to the $\mathrm{O}^{+}$ions per unit volume due to Coulomb collisions. Above $4000 \mathrm{~km}$ altitude, the gravitational and electric field forces represent the two largest terms on the RHS of Eq. (16) in all our four cases. For the time being, let us focus only on cases (b), (c), and (d) at these altitudes. As we can infer from Fig. 17, $e E-m_{o} g$ becomes more positive (or less negative) from cases (b) to (d). Thus, the LHS of Eq. (16) should increase from (b) to (d). Moreover, the $\mathrm{O}^{+}$density scale heights are very similar in these three cases, as the lines for $n_{o}$ are almost parallel in Fig. 15. Because $T_{o \|}$ in these cases is also within the same order at around $4000 \mathrm{~km}$ altitude, $\partial T_{o \|} / \partial s$ should increase from cases (b) through (d) according to Eq. (16). This is consistent with what Fig. 21 indicates: 

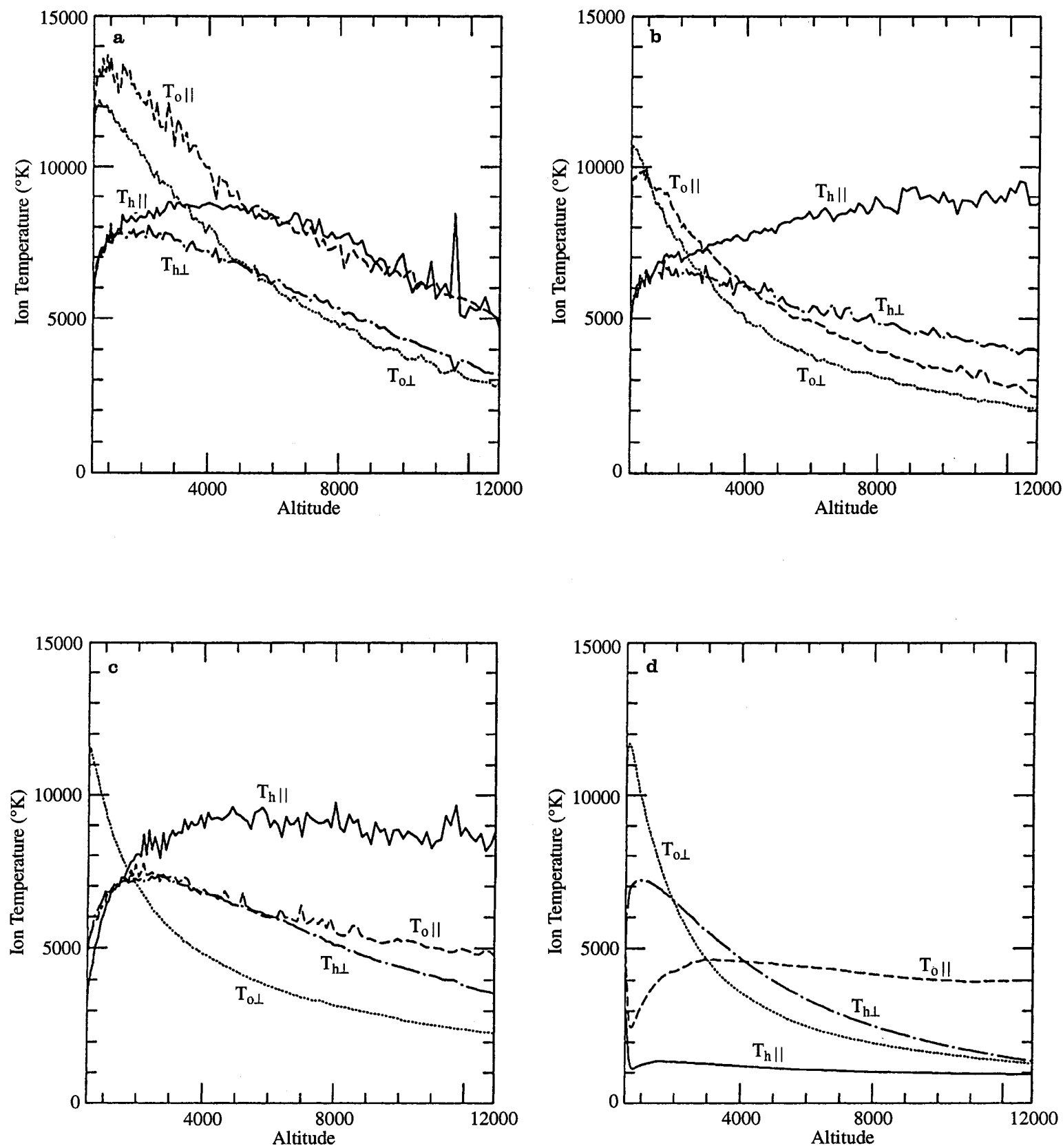

Fig. 21. Profiles of the ion temperatures. The labels correspond to the cases listed in Table 3

the gradient for $T_{o \|}$ becomes less negative from (b) through $(d)$.

There are also other effects in the polar wind that may contribute to the resulting ion temperature profiles. These effects, like diffusion and velocity-differential momentum transfer, modify the distributions of the species as the particles move along the field line. The temperatures at high altitudes are thus the integrated results of all the effects that govern the evolution of the particle distributions. A well-known effect is the mirror force that converts the particles' energies transverse to the field into the parallel direction as they travel upward. As the collisional effects weaken with altitude due to the density decrease, the mirror effect dominates the perpendicular temperature profiles at high altitudes, leading to downward gradients in $T_{h \perp}$ and $T_{o \perp}$ in all the cases considered.

\subsection{Discussion}

By varying the density ratio of the photoelectrons to the thermal electrons at the entry boundary, we have examined by comparison how and to what extent the day-night transition may affect the polar wind dynamics. The boundary conditions that we have used are within the experimental limits. However, even within such physical constraints, our polar wind solutions still demonstrate different qualitative features. Such qualitative differences are an indication of the complexity of the 
polar wind dynamics, an interplay between a number of physical effects. The impact of these effects on the plasma outflow depends on the polar wind quantities themselves, whose variations, therefore, may enhance or reduce these effects to different extents. Because some of these effects have opposite influences on certain quantities, the qualitative features of the polar wind strongly depend on which effect being the dominant factor. As we have shown, some of these physical effects in the polar wind are particularly sensitive to the transition between day and night. Thus, significantly different outflow properties may result due to this transition. Sometimes, the difference is so large that our polar wind solutions might even seem unrealistic. An example is the sharp electron temperature gradient at low altitudes as shown in Fig. 12(d). In that calculation, a relatively high density ratio is used for $n_{s} / n_{e}$ in the boundary condition. Although the density ratio has assumed a reasonable value, when it combines with the other realistic boundary conditions, the solution features results that may be beyond experimental limits. However, this does not mean that the density ratio itself is unrealistic; it only means the density ratio does not match well with the other boundary conditions.

It is worth reminding the reader that the boundary conditions in the kinetic part of our calculation are in fact distributions. Although these boundary distributions characterize a few moments, such as the density, mean velocity, and temperature of the species, they in fact possess an infinity of degrees of freedom, and should only be viewed as approximations to the boundary conditions of the solution. In this sense, the boundary conditions that we start with initially are based on educated guesses. The difference between these approximations and the exact boundary distributions may lead to some spurious manifestations (e.g., an exaggerated increase in ion temperature near the boundary). We are trying to develop a systematic procedure that will allow us to reduce these effects, and improve our comparisons with observations. In the present parametric study, we are primarily interested in how the transition between day and night affects the polar wind. However, we are also interested in how sensitive the dynamics of the outflow is to this transition. Thus, cases leading to exaggerated behavior near the lowaltitude boundary mentioned earlier are still useful in this regard.

\section{Conclusion}

Our work on the polar wind is motivated by the increasing experimental evidence that photoelectrons may affect the dynamics of the polar wind. In order to study the impact of photoelectrons on the polar wind, we rely on a self-consistent hybrid model that provides a global kinetic collisional description for the polar wind photoelectrons. The model is hybrid in that it consists of a fluid part for the thermal electrons and a kinetic collisional part, and should be distinguished from other hybrid schemes where, for example, the electrons are treated as a massless neutralizing fluid. Specifically, in this model, photoelectrons (which are treated as test particles because of their low relative density), and all the ion species $\left(\mathrm{H}^{+}, \mathrm{O}^{+}\right)$are described by a global kinetic collisional approach, while thermal electron properties and the ambipolar electric field are determined by a fluid calculation. The model is based on an iterative scheme: a physically meaningful solution is obtained when it converges. This model is the first to incorporate successfully the global kinetic collisional photoelectron description into a self-consistent polar wind picture. In addition, because of its kinetic treatment of the ions, it provides the first global steady-state polar wind calculation that generates a self-consistent continuous solution which varies from a low-altitude collisional subsonic regime to a high-altitude collisionless supersonic regime. Results from the calculations are quantitatively similar to the Akebono satellite observations in a variety of aspects. In fact, the self-consistency of the model lends additional credibility to our results in that they no longer rely on arbitrary choices of the electric field, which may possibly be the most important governing mechanism in the polar wind. In our results, the electric potential drop is consistent with the experimentally deduced values. The ion properties such as densities, outflow velocities and temperatures are also within experimental limits. An anisotropy between upwardly and downwardly going electrons, and an upwardly directed electron heat flux, both observed in the polar wind, also appear in our results.

We have performed a comparative study by varying the relative photoelectron density at the lower boundary (the ionosphere) in order to understand the qualitative polar wind features that characterize the day-night transition. Results of the study reveal the importance of kinetic effects and the intrinsic complexity of the polar wind dynamics. These results also demonstrate that a self-consistent description is indeed required for the comparative study. This is particularly true because some polar wind outflow properties are governed by competing effects, which are to different extents affected by these outflow properties themselves; only a selfconsistent description can take all these inter-relations into account.

Despite all these complex inter-relations, we find that an increase in the relative photoelectron density (corresponding to a decrease in the solar zenith angle, or a transition from night to day) enhances the self-consistent ambipolar electric field in the polar wind. In fact, our results have shown that these two quantities, together with the electron temperature, are all related. A higher relative photoelectron density at the boundary corresponds to a larger intrinsic electron heat flux. That leads to a larger ambipolar electric field. A larger portion of photoelectrons also means that the thermal electrons are more effectively heated through the collisions between the two electron components, because a greater heat source is available for each thermal electron on average. That leads to a higher thermal electron temperature, and a higher overall electron temperature. A higher temperature would lead to a larger pressure 
gradient. To balance that, a larger electric field is required.

The electric field enhancement associated with an increase in the relative photoelectron density seems to dictate the variation of other polar wind quantities, e.g. enhancement of the escaping ion flux, increase in the ion outflow velocities. In particular, the ion outflow velocity variation is consistent with the day-night asymmetry observed by the Akebono satellite. The electric field enhancement, however, leads to a more complicated variation in the ion densities. Intuitively, one would expect the ion density at high altitudes to increase with the electric field, because the field pushes more ions to escape. In fact, this is not the only effect of the electric field on the ion densities. Acceleration of the escaping ions will deplete the density of the species due to particle conservation. Thus, the electric field itself, through its acceleration of the ions, can create competing effects on the ion densities. We find that in general, when the relative photoelectron density is sufficiently low, (and hence the electric field is also sufficiently low), an increase in this quantity, which causes the electric field also to increase, leads to an increase in the ion densities. But this variation holds only up to a certain limit. Beyond that, an increase in the relative photoelectron density (which still causes the electric field to increase) causes the density to respond in an opposite way as the depletion effect takes over.

Coulomb collisions also play an important role in the polar wind dynamics. For example, the $\mathrm{H}^{+}-\mathrm{O}^{+}$collisions give rise to a slowing-down effect on the $\mathrm{H}^{+}$ions. This collisional effect competes against the acceleration by the electric field in governing the $\mathrm{H}^{+}$outflow velocity. The relative importance of this slowing-down effect is related to the $\mathrm{O}^{+}$density, due to the density dependence of the collisional rates. When the relative photoelectron density is sufficiently low such that an increase in the quantity will cause the ion densities to increase (see earlier), the slowing-down effect on the $\mathrm{H}^{+}$may be enhanced to a greater extent than the acceleration effect by the electric field.

This method, with its kinetic treatment of the ions, has enabled us to disentangle the competing physical effects governing the photoelectron-driven polar wind, and thus gain further insight into one of the important ionospheric-magnetospheric coupling mechanisms. In future work, we will extend its applicability by including wave-particle interaction, which should make it useful in the study of other important geoplasma settings, and develop a systematic procedure for the choice of boundary conditions, which should facilitate its use in parametric studies.

Acknowledgement: The authors would like to thank J.R. Jasperse, J.D. Winningham, Andrew Yau, and Takumi Abe for many useful discussions, John Retterer for participating in the initial stage of the investigation, and Supriya Ganguli for constant collaboration. This work is supported by NSF Grant ATM-9634599, AFOSR Grants F49620-93-1-0287 and F49620-96-1-0340, NASA Grant NAG5-2255, and AF Contract F19628-91-K-0043. Portion of this work was performed at the International Space Science Institute, Bern, Switzerland. T.C. and F.Y. wish to thank Professors
B. Hultqvist and J. Geiss for their kind hospitality.

Topical Editor D. Alcaydé thanks P.-L. Blelly for his help in evaluating this paper.

\section{References}

Abe, T., B. A. Whalen, A. W. Yau, R. E. Horita, S. Watanabe, and E. Sagawa, EXOS D (Akebono) suprathermal mass spectrometer observations of the polar wind, J. Geophys. Res., 98, 11191, 1993a.

Abe, T., B. A. Whalen, A. W. Yau, S. Watanabe, E. Sagawa, and K. I. Oyama, Altitude profile of the polar wind velocity and its relationship to ionospheric conditions, Geophys. Res. Lett., 20, $2825,1993 \mathrm{~b}$.

Abe, T., B. A. Whalen, A. W. Yau, E. Sagawa, and S. Watanabe, Akebono observations of thermal ion outflow and electron temperature in the polar wind region, in Physics of Space Plasmas (1995), Ed T. Chang, and J. R Jasperse, 14, p. 3, Cambridge, MA. MIT Center for Theoretical Geo/Cosmo Plasma Physics, 1996.

Axford, W. I., The polar wind and the terrestrial helium budget, $J$. Geophys. Res., 73, 6855, 1968.

Banks, P. M., and T. E. Holzer, The polar wind, J. Geophys. Res., 73, 6846, 1968.

Barakat, A. R., and R. W. Schunk, $\mathrm{O}^{+}$ions in the polar wind, $J$. Geophys. Res., 88, 7887, 1983.

Blelly, P. L., and R. W. Schunk, A comparative study of the timedependent standard 8-, 13- and 16-moment transport formulations of the polar wind, Ann. Geophysicae, 11, 443, 1993.

Book, D. L., Plasma physics, in A physicist's desk reference, Ed H. L. Anderson, chap. 18, p. 282. American Institute of Physics, New York, 2nd edn., 1989.

Chandler, M. O., Observations of downward moving $\mathrm{O}^{+}$in the polar topside ionosphere, J. Geophys. Res., 100, 5795, 1995.

Demars, H. G., and R. W. Schunk, Solutions to bi-Maxwellian transport equations for the polar wind, Planet. Space Sci., 37, $85,1989$.

Ganguli, S. B., The polar wind, Rev. Geophys., 34, 311, 1996.

Ganguli, S. B., H. G. Mitchell, Jr., and P. J. Palmadesso, Behavior of ionized plasma in the high latitude topside ionosphere: the polar wind, Planet. Space Sci., 35, 703, 1987.

Ichimaru, S., Plasma physics: an introduction to statistical physics of charged particles. Benjamin/Cummings, Menlo Park, CA, 1986.

Jasperse, J. R., The photoelectron distribution function in the terrestrial ionosphere, in Physics of space plasmas, Ed T. S. Chang, B. Coppi, and J. R. Jasperse, 4 in SPI Conference Proceedings and Reprint Series, p. 53, Cambridge, MA. Scientific Publishers, 1981.

Lee, J. S., J. P. Doering, T. A. Potemra, and L. H. Brace, Measurements of the ambient photoelectron spectrum from Atmosphere Explorer: II. AE-E measurements from 300 to $1000 \mathrm{~km}$ during solar minimum conditions, Planet. Space Sci., 28, 973, 1980.

Lemaire, J., Effect of escaping photoelectrons in a polar exospheric model, Space Res., 12, 1413, 1972.

Olbert, S., Role of thermal conduction in the acceleration of the solar wind, NASA Conf. Publ., p. 149, 1982.

Parker, E. N., Dynamics of the interplanetary gas and magnetic fields. Astrophys. J., 128, 664, 1958.

Raitt, W. J., R. W. Schunk, and P. M. Banks, A comparison of the temperature and density structure in high and low speed thermal proton flows, Planet. Space Sci., 23, 1103, 1975.

Retterer, J. M., T. Chang, G. B. Crew, J. R. Jasperse, and J. D. Winningham, Monte Carlo modeling of ionospheric oxygen acceleration by cyclotron resonance with broad-band electromagnetic turbulence, Phys. Rev. Lett., 59, 148, 1987.

Schunk, R. W., Mathematical structure of transport equations for multispecies flows, Res. Geophys. Space Phys., 15, 429, 1977. 
Schunk, R. W., and D. S. Watkins, EIectron temperature anisotropy in the polar wind, J. Geophys. Res., 86, 91, 1981.

Scudder, J. D., and S. Olbert, A theory of local and global processes which affect solar wind electrons: 1. the origin of typical 1 AU velocity distribution functions - steady state theory, J. Geophys. Res., 84, 2755, 1979.

Tam, S. W. Y., F. Yasseen, T. Chang, and S. B. Ganguli, Selfconsistent kinetic photoelectron effects on the polar wind, Geophys. Res. Lett., 22, 2107, 1995a.

Tam, S. W. Y., F. Yasseen, T. Chang, S. B. Ganguli, and J. M. Retterer, Anisotropic kinetic effects of photoelectrons on polar wind transport, in Cross-scale coupling in space plasmas, Ed J. L. Horwitz, N. Singh, and J. L. Burch, 93 in Geophysical Monograph, p. 133, Washington D.C. American Geophysical Union, 1995b.
Winningham, J. D., and C. Gurgiolo, DE-2 photoelectron measurements consistent with a large scale parallel electric field over the polar cap, Geophys. Res. Lett., 9, 977, 1982.

Winningham, J. D., and W. J. Heikkila, Polar cap auroral electron fluxes observed with Isis 1, J. Geophys. Res., 79, 949, 1974.

Yasseen F., and J. M. Retterer, Critical points in the 16-moment approximation, J. Geophys. Res., 96, 1827, 1991.

Yasseen, F., J. M. Retterer, T. Chang, and J. D. Winningham, Monte-Carlo modeling of polar wind photoelectron distributions with anomalous heat flux, Geophys. Res. Lett., 16, 1023, 1989.

Yau, A. W., B. A. Whalen, T. Abe, T. Mukai, K. I. Oyama, and T. Chang, Akebono observations of electron temperature anisotropy in the polar wind, J. Geophys. Res., 100, 17451, 1995. 